\title{
Predicting Hepatocellular Carcinoma Risk in Patients with Chronic HCV Infection and a Sustained Virological Response to Direct-Acting Antivirals
}

\author{
Roberta D'Ambrosio' \\ Elisabetta Degasperi ${ }^{1}$ \\ Pietro Lampertico ${ }^{1,2}$ \\ 'Foundation IRCCS Ca' Granda Ospedale \\ Maggiore Policlinico, Division of \\ Gastroenterology and Hepatology, Milan, \\ Italy; ${ }^{2}$ CRC "A. M. and A. Migliavacca" \\ Center for Liver Disease, Department of \\ Pathophysiology and Transplantation, \\ University of Milan, Milan, Italy
}

\begin{abstract}
Chronic infection with hepatitis $\mathrm{C}$ virus (HCV) may complicate with hepatocellular carcinoma (HCC), especially in patients with cirrhosis. Although the achievement of a sustained virological response (SVR) had been associated with a reduction in the risk of HCC already in the Interferon era, some concerns initially raised following the use of directacting antivirals (DAA), as their use was associated with increased risk of HCC development and aggressiveness. However, studies demonstrated that the risk of HCC was strongly influenced by pre-treatment fibrosis stage and, eventually, prior HCC history more than the type of antiviral therapy. According to published studies, rates of de-novo HCC ranged between $1.4 \%$ and $13.6 \%$ in patients with cirrhosis or advanced fibrosis vs $0.9 \%$ and $5.9 \%$ in those with chronic hepatitis $\mathrm{C}$ (CHC). Conversely, rates of recurrent $\mathrm{HCC}$ were higher, ranging between $3.2 \%$ and $49 \%$ in cirrhotics vs $0 \%$ and $40 \%$ in $\mathrm{CHC}$ patients. Most studies tried to identify predictors of HCC development, either de-novo or recurrent, and some authors were also able to build predictive scores for HCC risk stratification, which however still need prospective validation. Whereas some clinical features, such as age, gender, presence of comorbidities and fibrosis stage, may influence both de-novo and recurrent HCC, previous tumour burden before DAA seems to prevail over these features in recurrent HCC risk prediction.
\end{abstract}

Keywords: hepatocellular carcinoma, $\mathrm{HCC}$, hepatitis $\mathrm{C}$ virus, $\mathrm{HCV}$, sustained virological response, SVR, direct-antiviral agent, DAA, surveillance, predictor

\section{Introduction}

Hepatocellular carcinoma (HCC) is currently the fourth cause of liver-related death worldwide, ${ }^{1,2}$ and accounts for one of the most frequent indications for liver transplantation. In patients with chronic hepatitis $\mathrm{C}(\mathrm{CHC})$, the achievement of a sustained virological response (SVR) to antiviral treatment was demonstrated to reduce the incidence of $\mathrm{HCC}$, already in the Interferon (IFN) era, ${ }^{3-6}$ with a more pronounced benefit in those with advanced fibrosis or cirrhosis. After direct-acting antivirals (DAA) approval, a first pivotal study suggested a time-related association between DAA treatment and HCC recurrence, ${ }^{7}$ this finding being initially supported by others. ${ }^{8,9}$ Similarly, some authors also reported an increased incidence and biological aggressiveness of de-novo HCC arising in cirrhotics successfully treated with DAA. ${ }^{9-11}$ Next, evidences eventually raised against a definite role of oral antihepatitis $\mathrm{C}$ virus (HCV) treatments as HCC promoter. ${ }^{12-19}$ Different crude incidences of HCC in IFN vs DAA-treated cirrhotics mostly rely on differences in
Correspondence: Roberta D'Ambrosio Division of Gastroenterology and

Hepatology, Foundation IRCCS Ca' Granda Ospedale Maggiore Policlinico, Milan, Italy

Tel +39-0255035432

$\mathrm{Fax}+39-0250320410$

Email roberta.dambrosio@policlinico.mi.it 
patient population, as DAA allow treatment of patients with more advanced liver diseases. Following an SVR to DAA-based regimens, reported rates of de-novo HCC are estimated nearly $2-2.5 \%{ }^{17,20-22}$ vs $20-30 \%$ per year $^{18,19,23}$ of recurrent HCC, being definitively higher than that historically reported in the setting of IFN. ${ }^{4}$ Moreover, HCC risk has been demonstrated to persist up to 10 years from treatment completion. $^{17}$

Taking together, these data still justify the need for long-life surveillance, ${ }^{1,24}$ resulting in intensive follow-up of large cohorts of cured HCV patients. Therefore, current literature efforts aim at deeply investigating predictors of HCC in HCV patients cured through SVR, with the ultimate goal of personalized risk stratification and individualized surveillance policies.

Therefore, in this review, we report data from published study analyzing the risk of HCC development, either de-novo or recurrent, following DAA-based treatments. Particularly, we focused on those studies reporting not only full patients' characteristics but also information on $\mathrm{HCC}$ rates and predictors.

\section{Predictors of de-novo HCC}

According to published studies, up to $14 \%$ of patients without history of previous liver cancer may develop denovo $\mathrm{HCC}$ after $\mathrm{HCV}$ eradication, although data vary according to patient population, follow-up duration and severity of liver disease (Table 1).

\section{Studies Enrolling Patients with Cirrhosis or Advanced Fibrosis}

Among 14 studies that reported data in cirrhotic patients, ${ }^{8,10,20-22,25-37}$ (Table 2), most were able to identify HCC predictors (Table 3). In addition, 5 studies enrolling patients with $\mathrm{CHC}$ and any fibrosis stage also found-out factors associated with de-novo $\mathrm{HCC}$ in the subset of patients with cirrhosis ${ }^{38-42}$ although unable to provide their clinical features (Tables 4 and 5). Finally, 5 authors reported data on patients with advanced fibrosis, defined through histology (F3-F4), non-invasive tests or criteria for chronic advanced liver disease (cACLD) ${ }^{11,36,43-46}$ (Table 2). Eight out of these studies enrolled only patients with an SVR, ${ }^{10,21,30,33,37,44-46}$ whilst in one study data could be extrapolated. ${ }^{34}$ In studies including also non-SVR patients, rates of treatment failure ranged between $1.9 \%$ and $10 \%$ (Table 2). Follow-up duration varied according to
Table I Assessment of Liver Fibrosis Severity According to Studies' Designs

\begin{tabular}{|c|c|c|}
\hline $\begin{array}{l}\text { Liver } \\
\text { Disease } \\
\text { Severity }\end{array}$ & $\begin{array}{l}\text { Tool for } \\
\text { Staging }\end{array}$ & Authors \\
\hline \multicolumn{3}{|l|}{ Cirrhosis } \\
\hline Histology & METAVIR F4 & 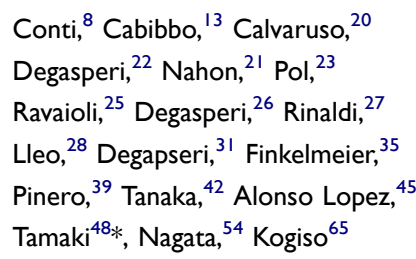 \\
\hline Clinical & $\begin{array}{l}\text { Any clinical } \\
\text { features } \\
\text { US features }\end{array}$ & $\begin{array}{l}\text { Cabibbo, }{ }^{13} \text { Calvaruso, }{ }^{20} \text { Degasperi, } \\
\text { Ravaioli, }{ }^{25} \text { Rinaldi, }{ }^{27} \text { lleo, } \\
\text { Degasperi, }{ }^{26} \text { Degasperi, } \\
\text { Sangiovanni, }{ }^{32} \text { Ogawa, }{ }^{38} \text { Alonso } \\
{\text { Lopez, }{ }^{45} \text { Kwon, },{ }^{51} \text { Ogawa },{ }^{55} \text { Kogiso, }}^{65} \\
\text { Zou }^{67}\end{array}$ \\
\hline FIB-4 & $\begin{array}{l}>3.25 \\
\text { Not specified }\end{array}$ & $\begin{array}{l}\text { Tanaka, }{ }^{42} \text { Nagata }^{54} \\
\text { Ide }^{40}\end{array}$ \\
\hline LSM & $\begin{array}{l}\geq 12 \mathrm{kPa} \\
>12 \mathrm{kPa} \\
>12.5 \mathrm{kPa} \\
\geq 12.5 \mathrm{kPa} \\
\geq 13.5 \mathrm{kPa} \\
\geq 14.9 \mathrm{kPa} \\
>16.2 \mathrm{kPa} \\
\text { Not specified }\end{array}$ & 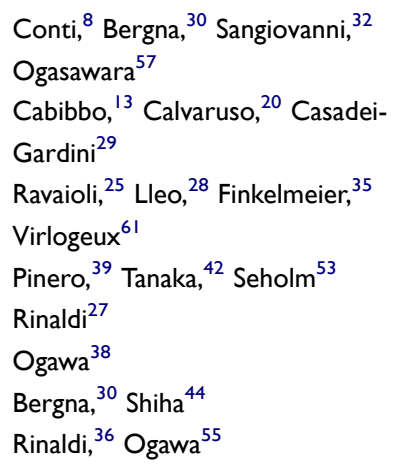 \\
\hline ICD codes & & Kanwal, ${ }^{34}$ Kanwal, $^{41}$ Zou $^{67}$ \\
\hline \multicolumn{3}{|c|}{ Advanced fibrosis } \\
\hline Histology & $\begin{array}{l}\text { METAVIR F3- } \\
\text { F4 }\end{array}$ & Nagata $^{54}$ \\
\hline LSM & $\begin{array}{l}>9.5 \mathrm{kPa} \\
>10 \mathrm{kPa} \\
\geq 10 \mathrm{kPa} \\
>10.2 \text { and } \\
\leq 16.2 \mathrm{kPa}\end{array}$ & $\begin{array}{l}\text { Pinero, }{ }^{39} \text { Alonso Lopez } \\
\text { Ogawa }^{48} \\
\text { Pons, }{ }^{46} \text { Seholm } \\
\text { Shiha }\end{array}$ \\
\hline FIB-4 & $>3.25$ & Tani, $^{52}$ Nagata, $^{54}$ Watanabe ${ }^{59}$ \\
\hline APRI & $\geq 1$ & Watanabe $e^{59}$ \\
\hline
\end{tabular}

Notes: *Available in 191 out of 346 patients.

Abbreviations: F, fibrosis; US, ultrasound; FIB-4, fibrosis-4 index; LSM, liver stiffness measurement; APRI, AST to platelet ratio; ICD, international classification of diseases (code).

study designs as reported in Table 2. Overall, studies reported de-novo HCC rates of $1.8-13.6 \%$ in cirrhotics, and of $1.4-4.6 \%$ in patients with advanced fibrosis. 


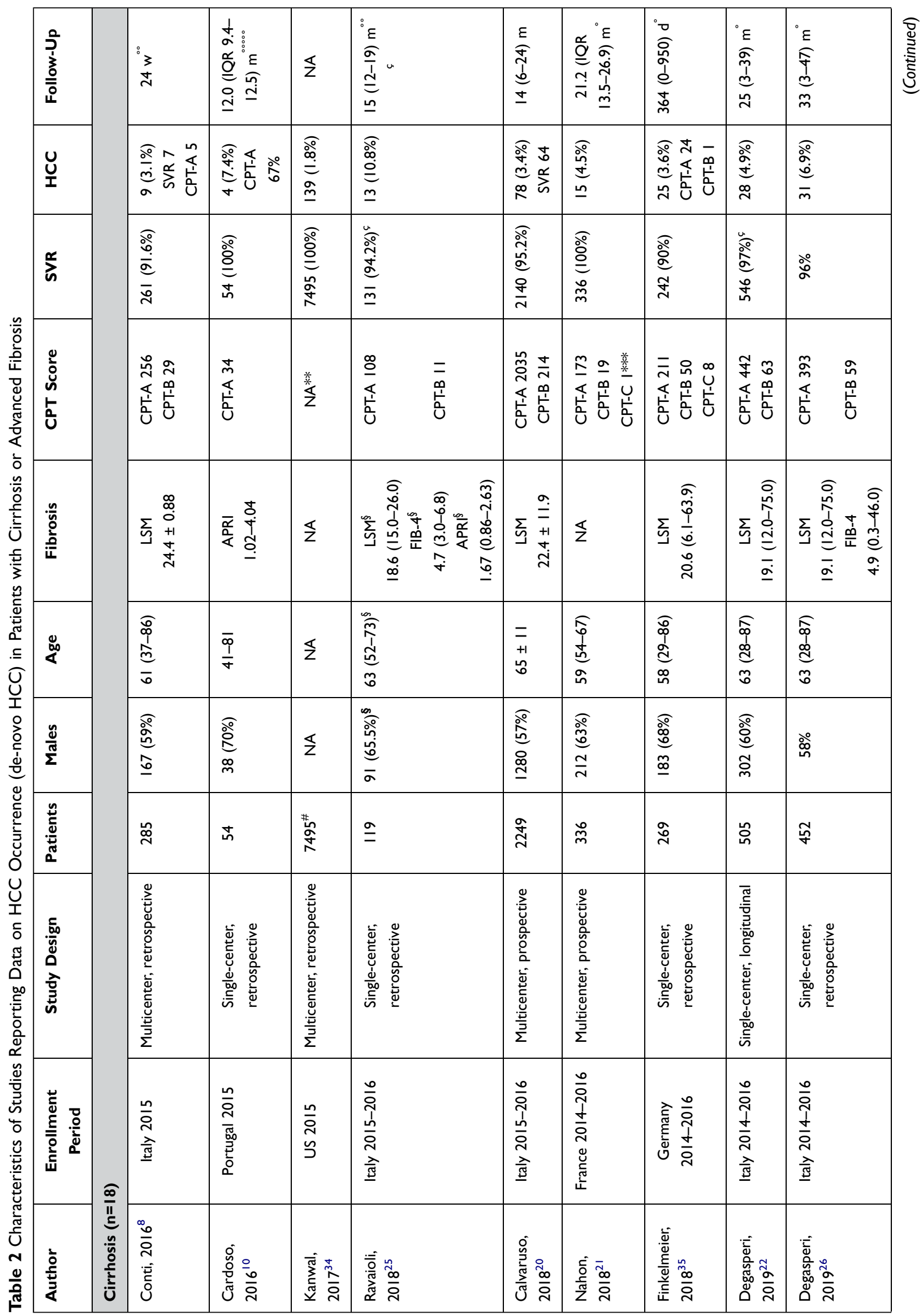




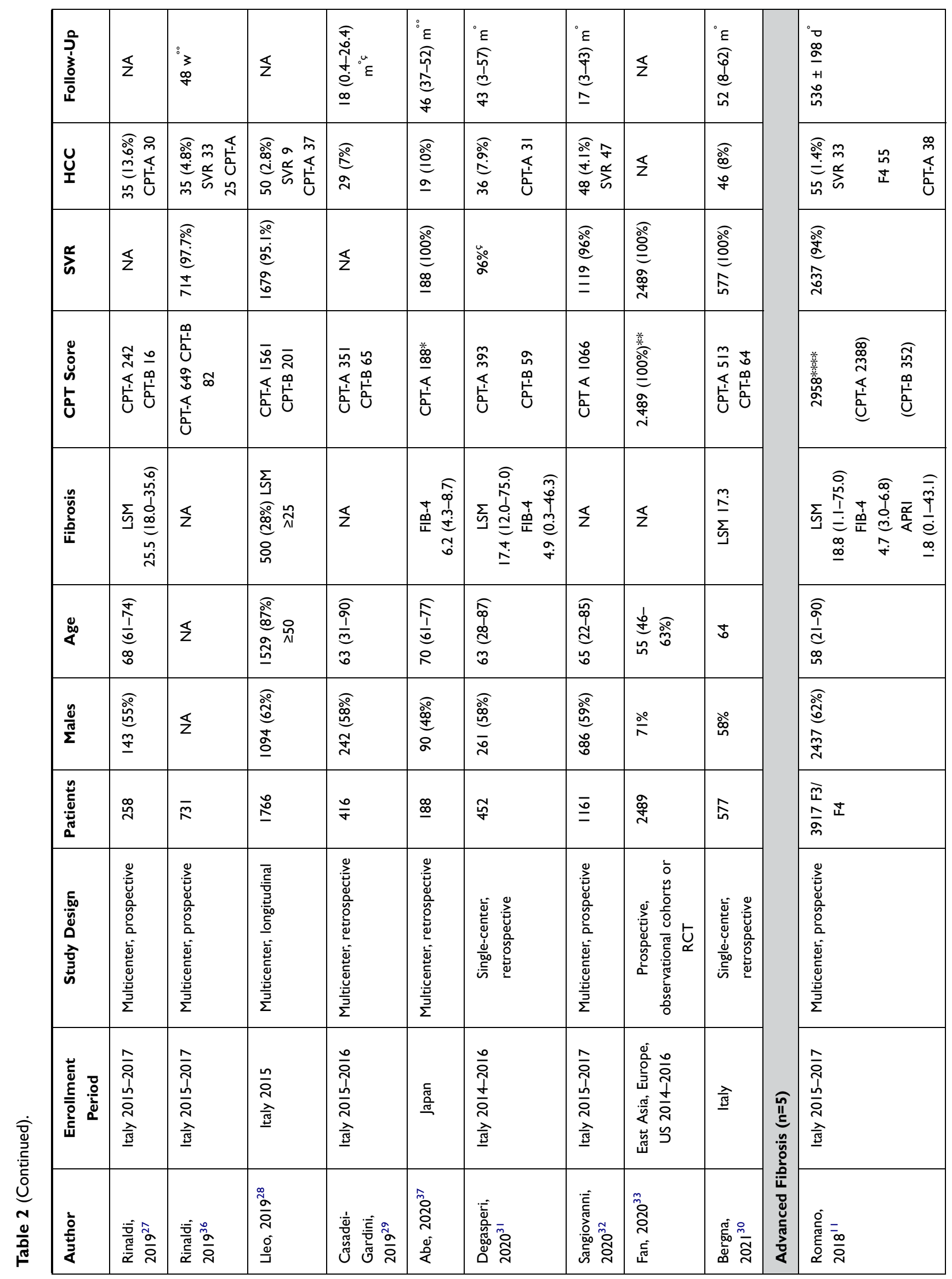




\begin{tabular}{|c|c|c|c|c|}
\hline \begin{tabular}{l}
$: 3$ \\
3 \\
$\infty$ \\
$\vdots$ \\
\hdashline
\end{tabular} & 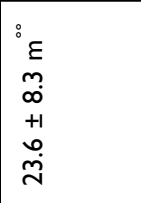 & 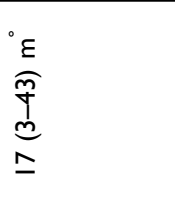 & 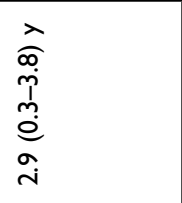 & \\
\hline 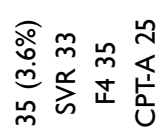 & 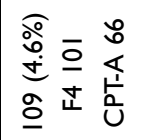 & 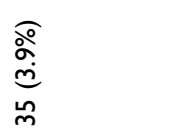 & 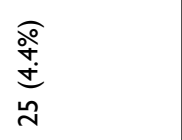 & \\
\hline $\begin{array}{l}\frac{0}{2} \\
\frac{0}{0} \\
\stackrel{0}{\circ} \\
\frac{0}{0}\end{array}$ & 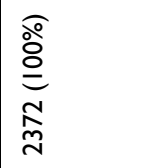 & $\frac{\text { ळo }}{\stackrel{0}{\circ}}$ & 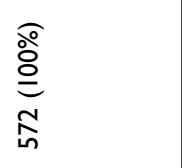 & \\
\hline 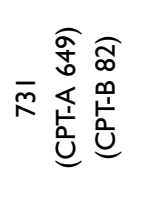 & 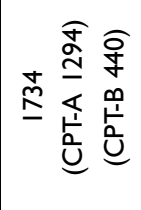 & 艺 & $\stackrel{*}{\mathrm{*}}$ & \\
\hline 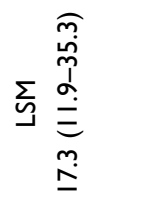 & $\S$ & 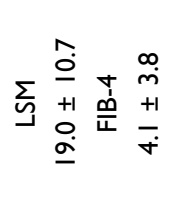 & 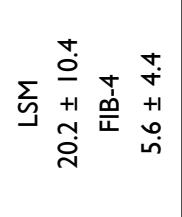 & \\
\hline $\begin{array}{l}\hat{\sigma} \\
\hat{1} \\
0 \\
\hat{n}\end{array}$ & 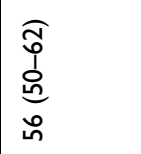 & 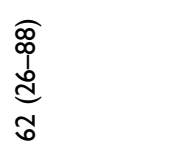 & $\begin{array}{l}= \\
+1 \\
+ \\
0\end{array}$ & \\
\hline $\begin{array}{l}\text { 号 } \\
\text { h్م } \\
\text { ơ }\end{array}$ & 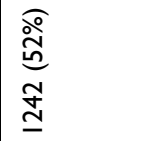 & 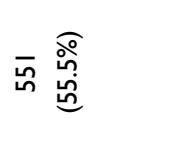 & 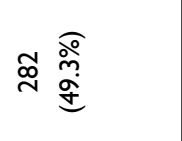 & \\
\hline 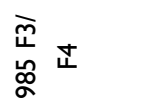 & $\underset{\substack{\tilde{M} \\
\sim}}{\mathbb{N}}$ & 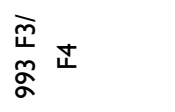 & 藏 & \\
\hline 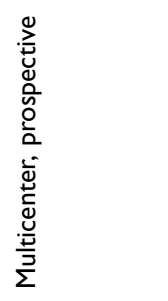 & 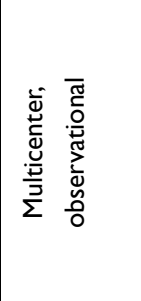 & 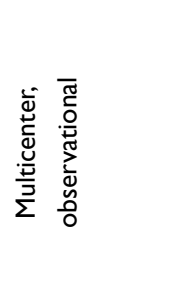 & 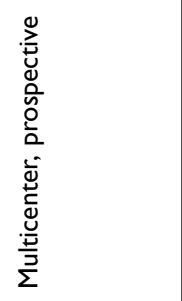 & \\
\hline 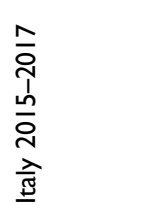 & 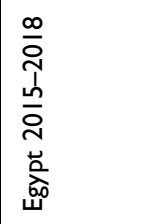 & 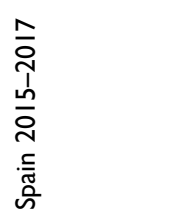 & 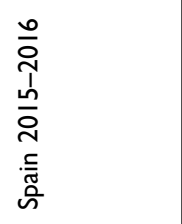 & \\
\hline 焥 & 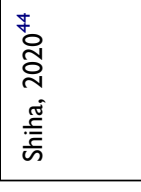 & 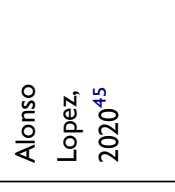 & 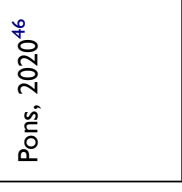 & \\
\hline
\end{tabular}




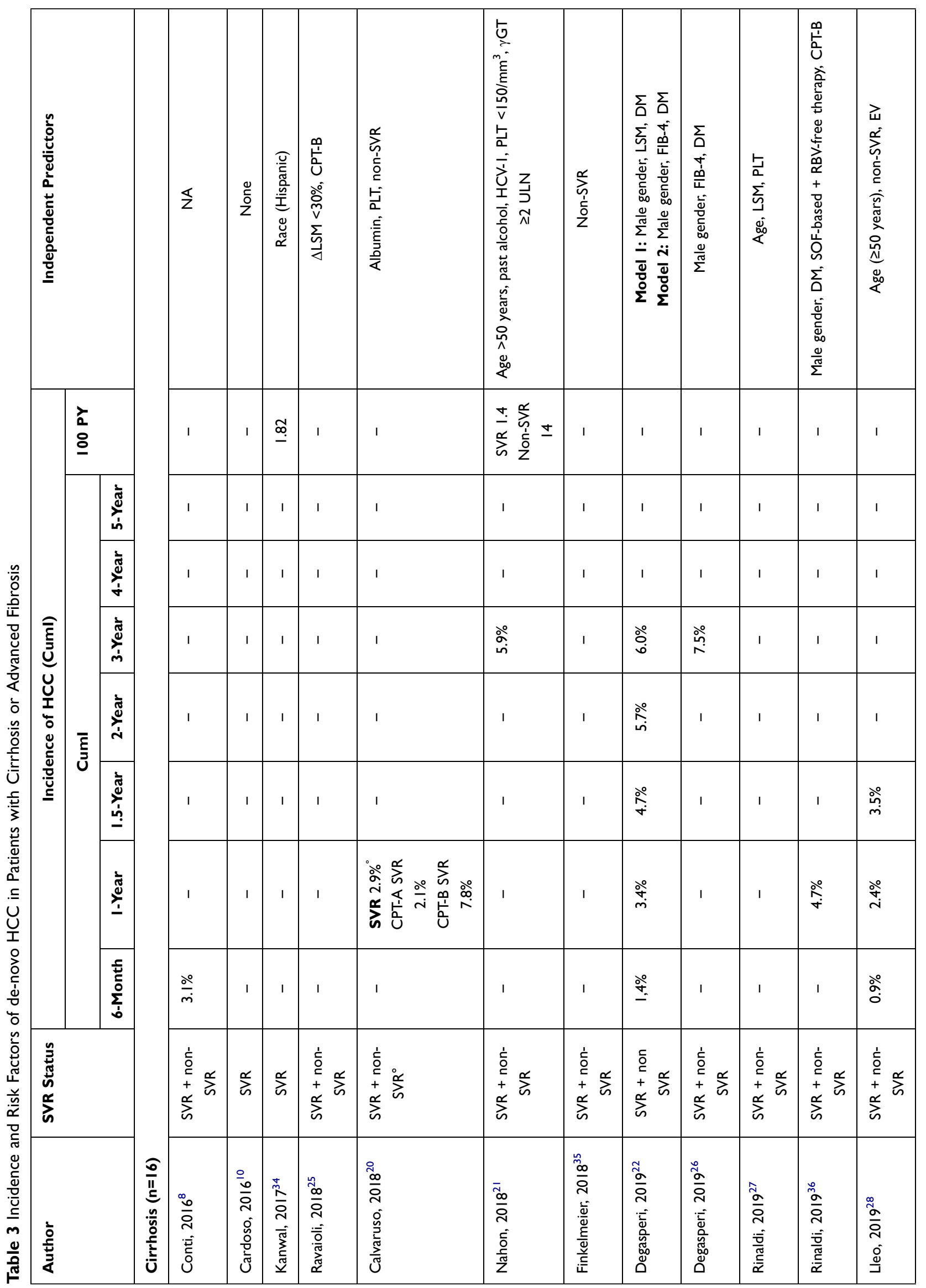




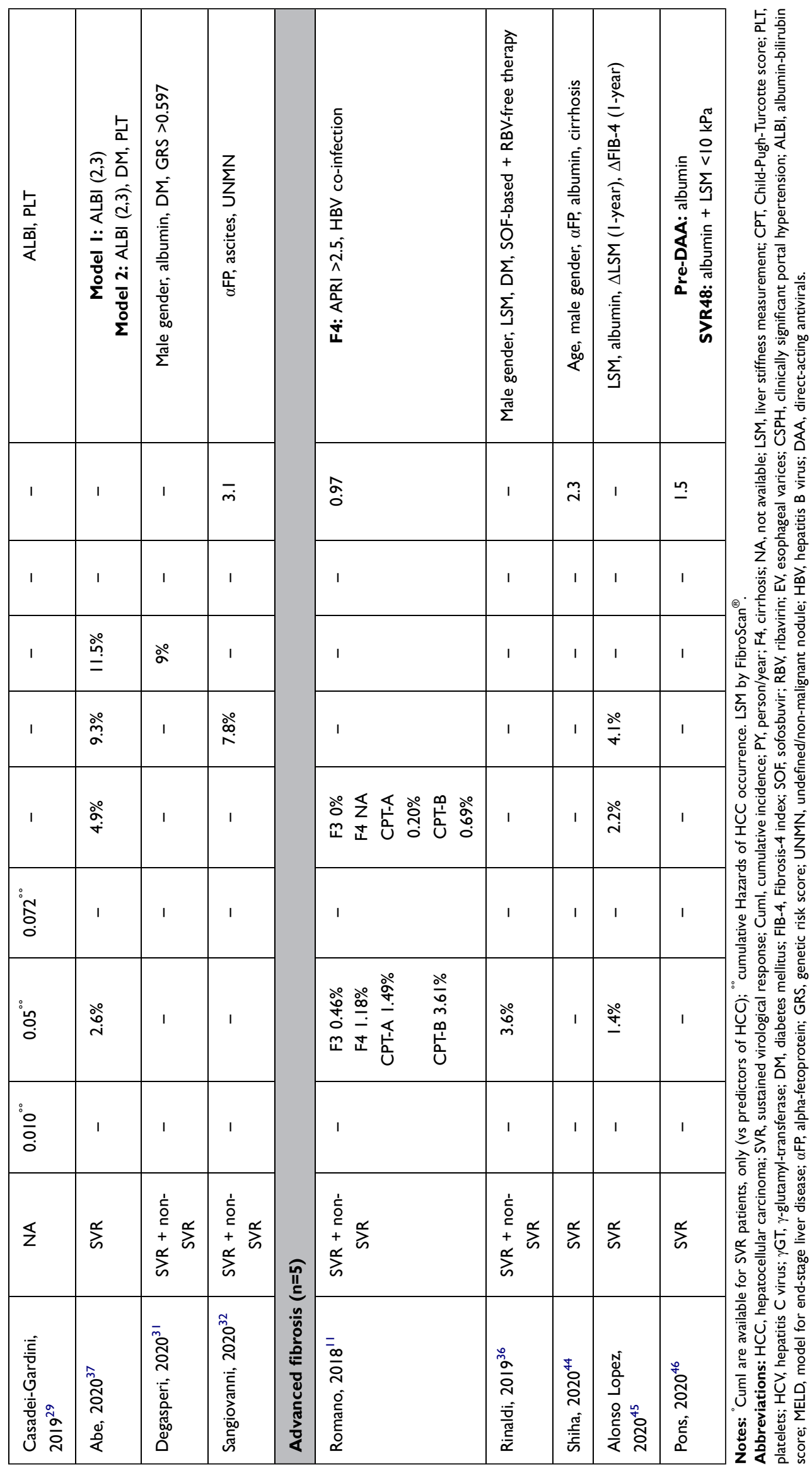




\begin{tabular}{|c|c|c|c|c|c|c|c|c|c|c|c|c|c|c|}
\hline 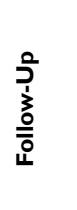 & 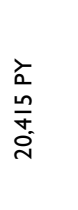 & 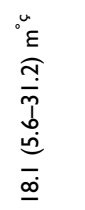 & 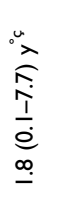 & $\begin{array}{l}\stackrel{0}{E} \\
\underset{I}{I} \\
\Sigma \\
\simeq\end{array}$ & 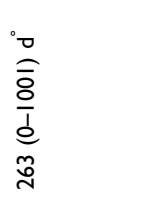 & 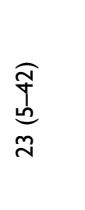 & $\begin{array}{l}8 \\
3 \\
\infty \\
8\end{array}$ & 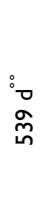 & $\begin{array}{l}\vdots \\
\varepsilon \\
\underline{n} \\
\alpha \\
+1 \\
m \\
\underline{0}\end{array}$ & 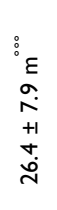 & 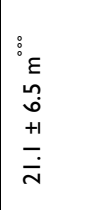 & $\begin{array}{l}0 \\
3 \\
0 \\
0\end{array}$ & 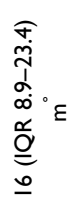 & 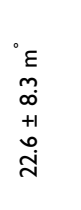 \\
\hline U⿺ & $\begin{array}{l}\stackrel{0}{\circ} \\
\stackrel{0}{\circ} \\
\underline{\infty}\end{array}$ & 兽 & 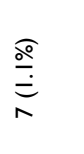 & 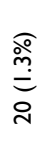 & 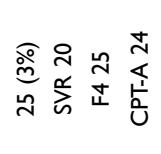 & $\frac{\substack{\circ \\
\stackrel{\circ}{\rho}}}{m}$ & $\begin{array}{l}\widehat{o} \\
\stackrel{0}{0} \\
\stackrel{0}{0} \\
\stackrel{m}{m}\end{array}$ & 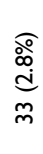 & 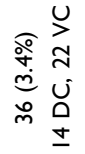 & 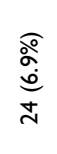 & $\begin{array}{l}\widehat{o} \\
\stackrel{\circ}{0} \\
\underline{\infty} \\
\underline{\omega}\end{array}$ & 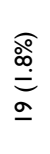 & 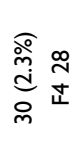 & 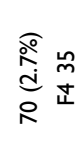 \\
\hline 站 & 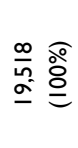 & 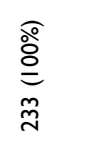 & 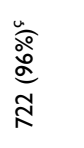 & 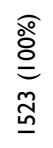 & 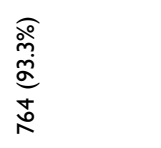 & $\mathbb{z}$ & $\begin{array}{l}\text { o̊ } \\
\stackrel{\circ}{\circ} \\
\text { ঃ }\end{array}$ & 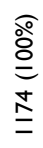 & 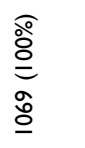 & 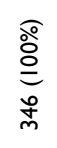 & 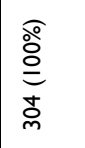 & 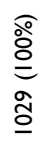 & 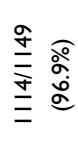 & 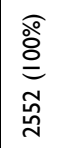 \\
\hline 亮 & 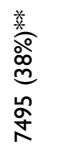 & $\frac{\text { 美 }}{z}$ & 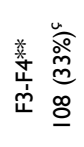 & 尊 & 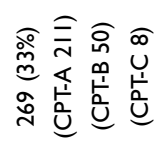 & 0 & $\frac{\widehat{\circ}}{\stackrel{0}{c}}$ & $\frac{1}{z}$ & $\frac{\text { 美 }}{z}$ & 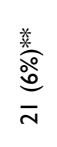 & $\frac{{ }_{3}^{*}}{z}$ & $\frac{*}{z}$ & 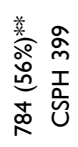 & 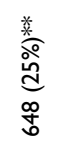 \\
\hline 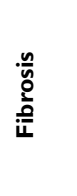 & $\mathbb{z}$ & 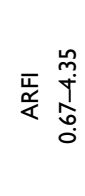 & 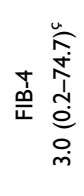 & $\underline{z}$ & 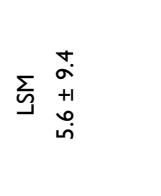 & 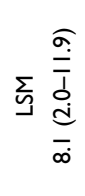 & 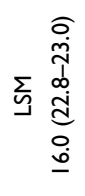 & $\mathbb{z}$ & 咅 & $\S$ & 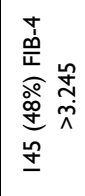 & $\mathbb{z}$ & $\underline{z}$ & 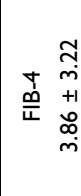 \\
\hline
\end{tabular}

要

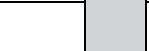

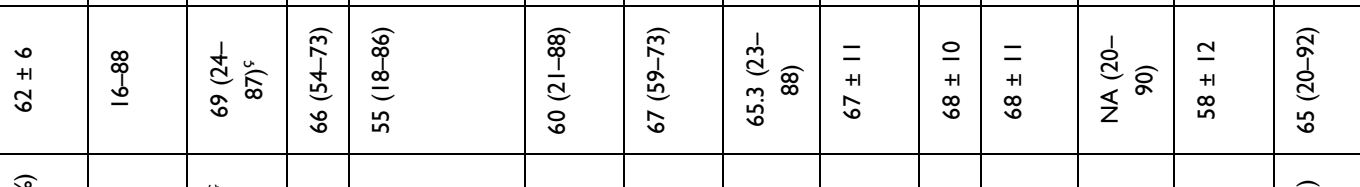

Uิ

o

$\frac{\sqrt{0}}{\frac{\pi}{\Sigma}}$

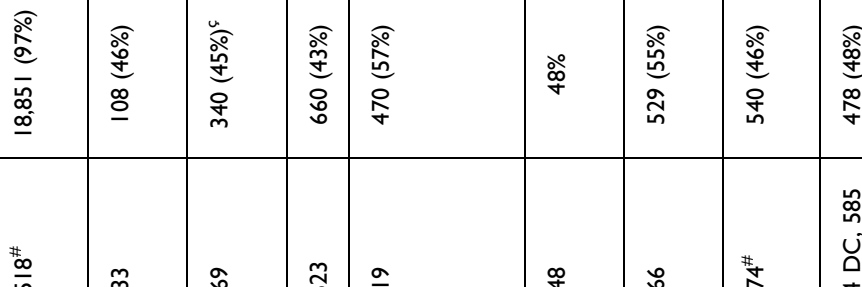

今ั

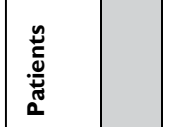

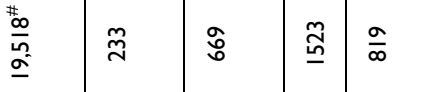

帝

บ

ธ

迎

$\stackrel{\circ}{\stackrel{c}{t}}$

$\stackrel{\circ}{\circ}$

$\stackrel{y}{\frac{\pi}{2}}$

芯

芩

竞

U

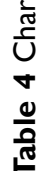

京

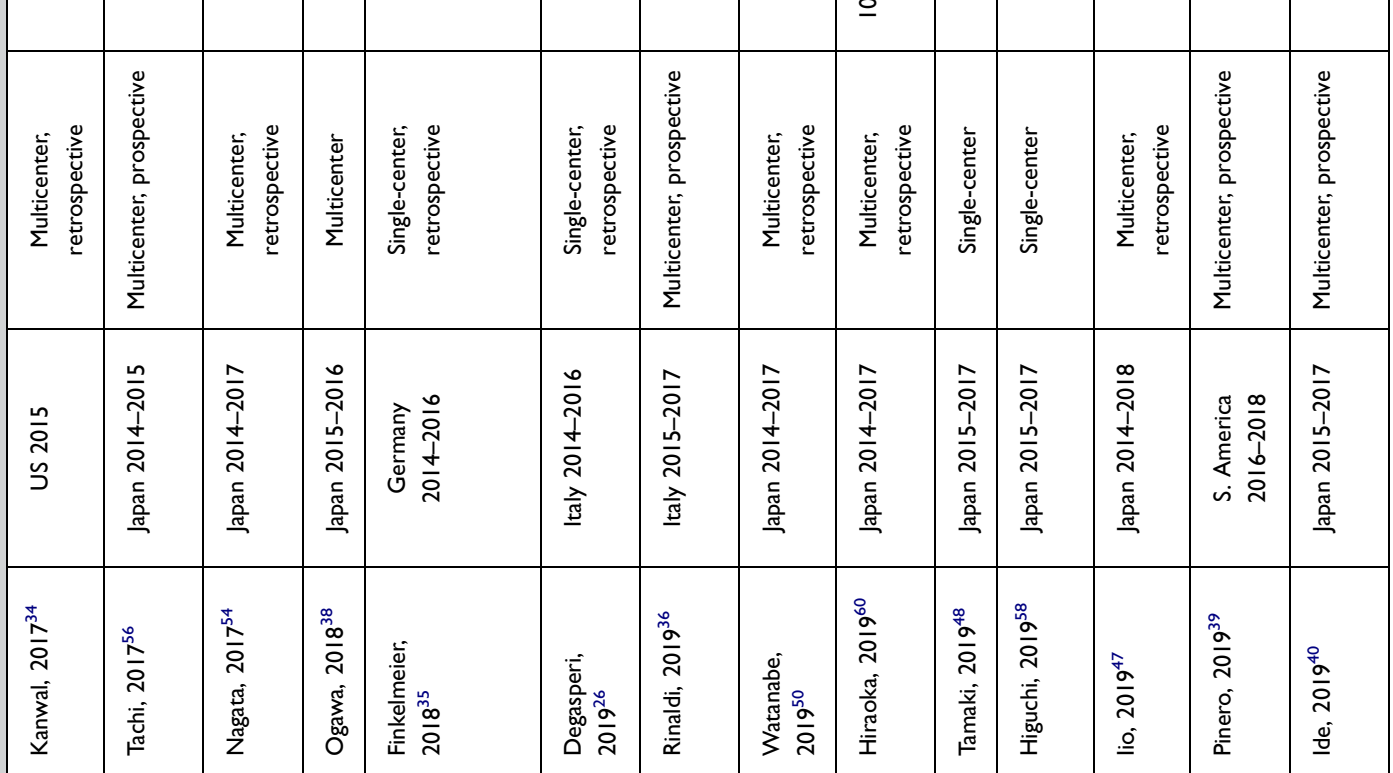




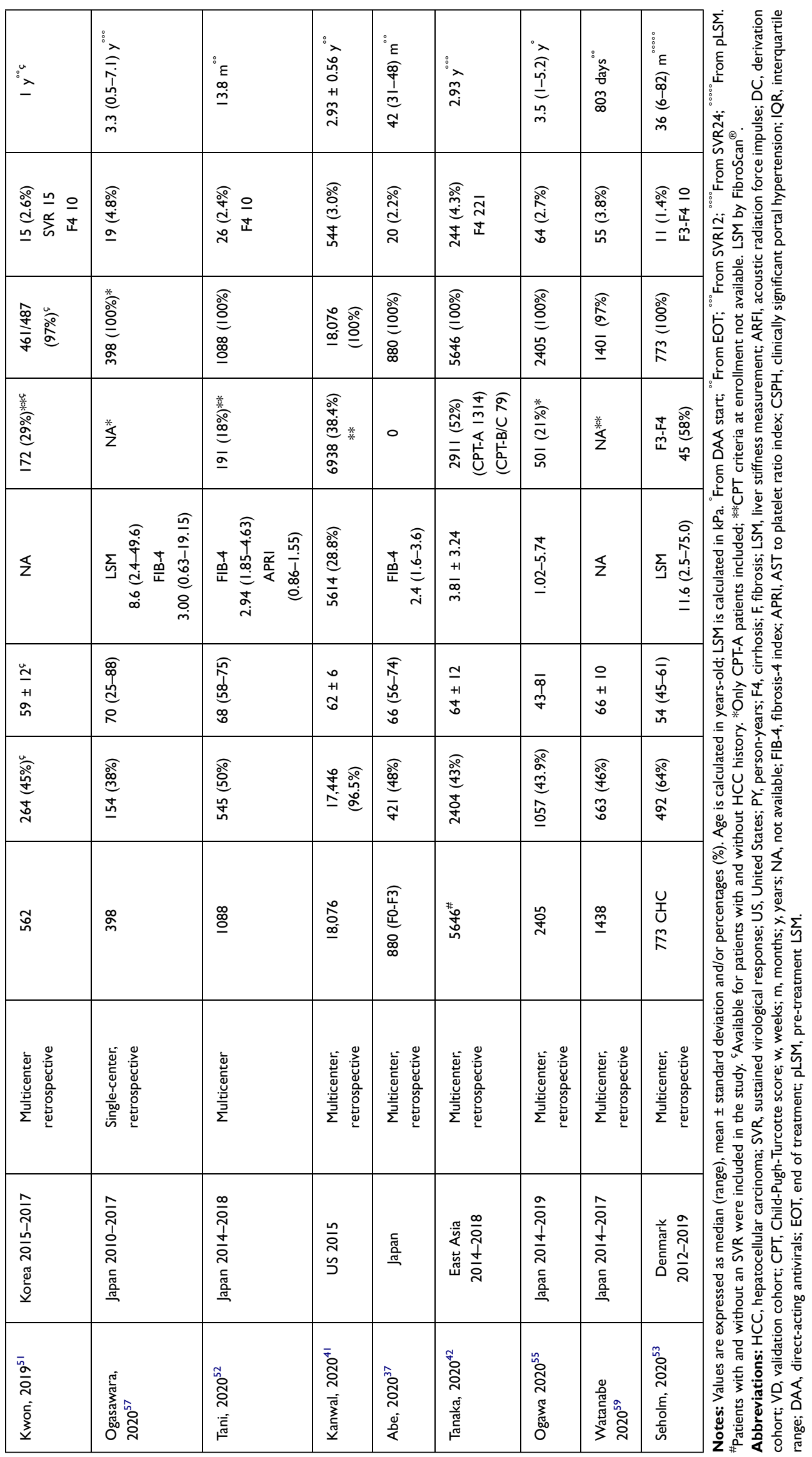




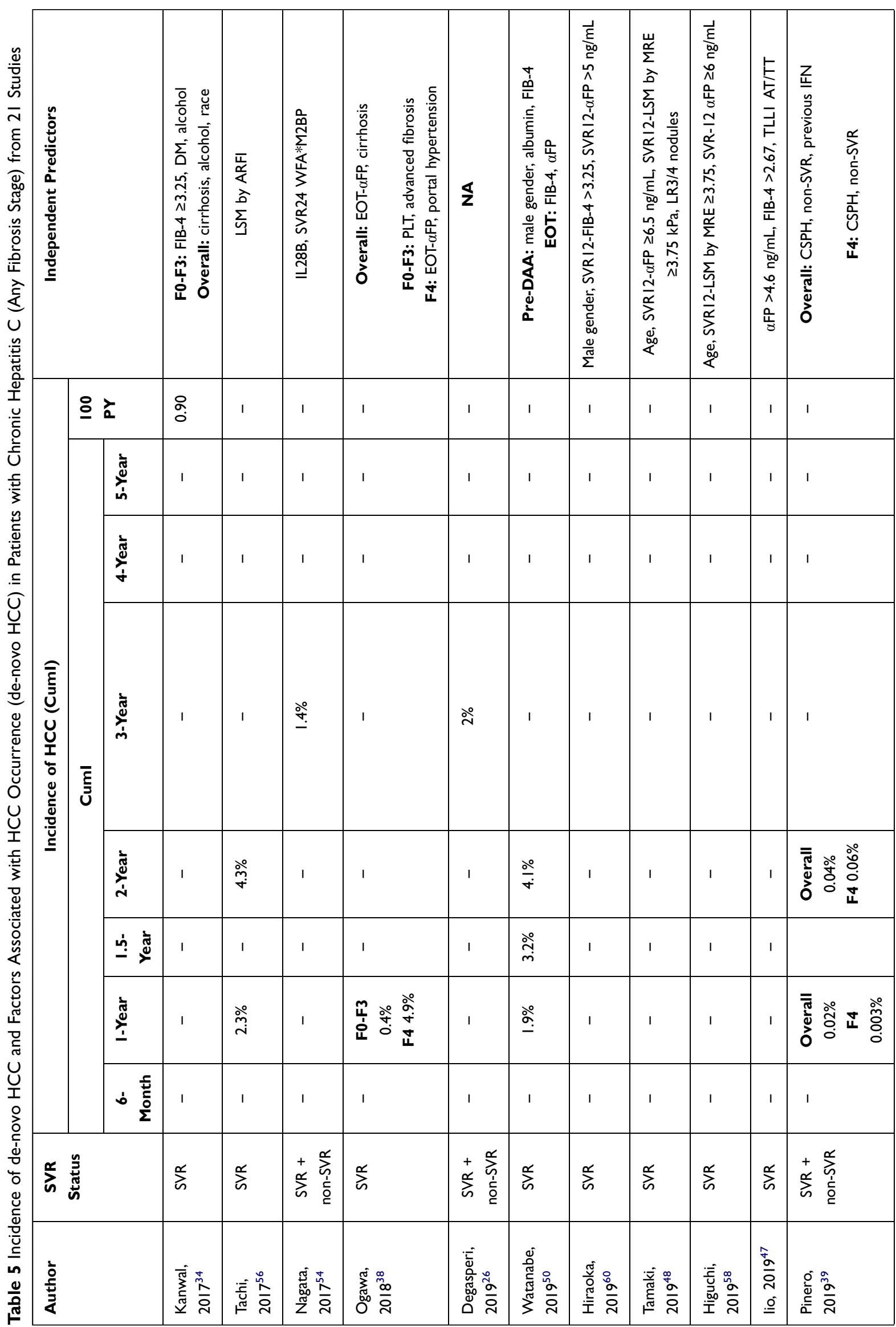




\begin{tabular}{|c|c|c|c|c|c|c|c|c|c|c|}
\hline 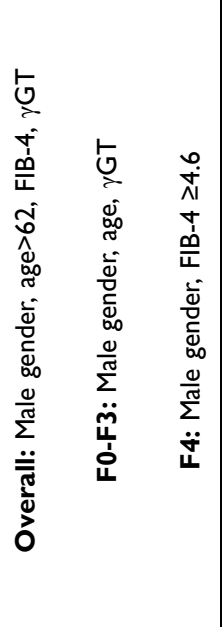 & 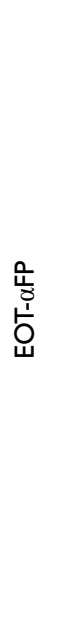 & 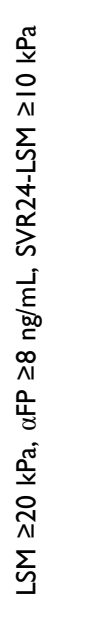 & 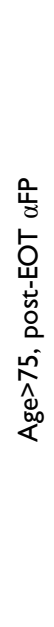 & 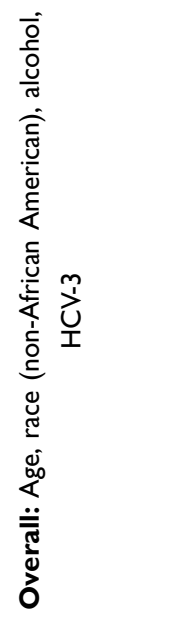 & 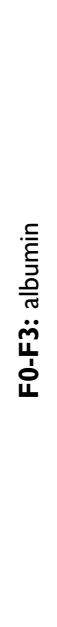 & 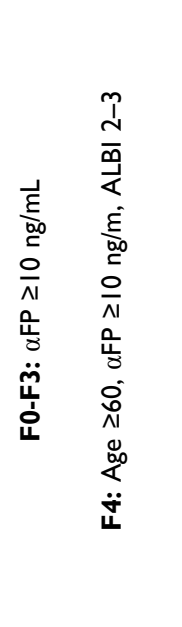 & 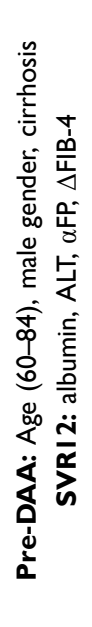 & 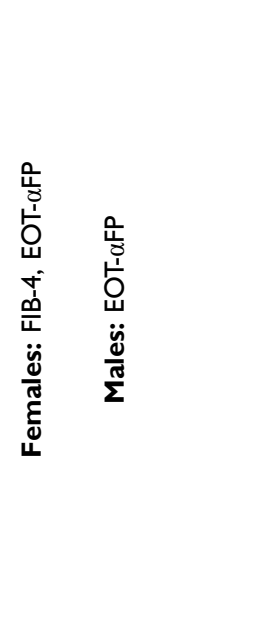 & 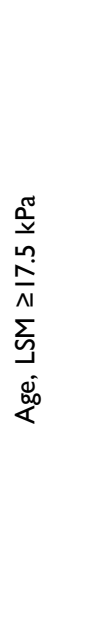 & 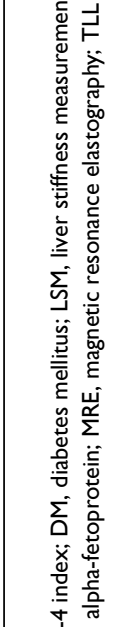 \\
\hline 1 & 1 & 1 & 1 & 1 & 1 & & 尚 & 1 & $\stackrel{\text { กn }}{0}$ & 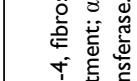 \\
\hline 1 & 1 & 1 & 1 & 1 & 1 & 苍 & 1 & 1 & 1 & 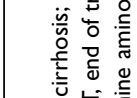 \\
\hline 1 & 1 & ợ & 1 & 1 & ळें & 1 & 1 & 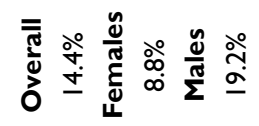 & 1 & 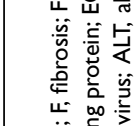 \\
\hline 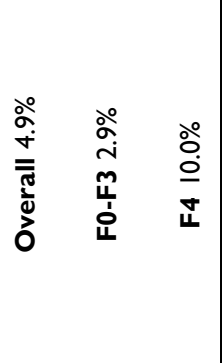 & 1 & 1 & ஃ̊ & 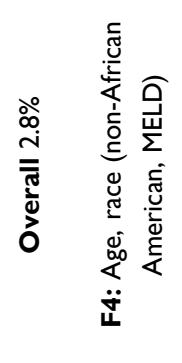 & ○े & 1 & 1 & 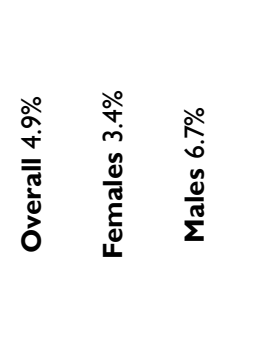 & 1 & 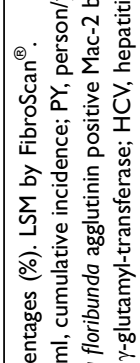 \\
\hline 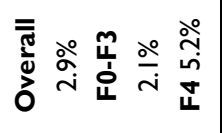 & 1 & ठ̊ำ & $\stackrel{\circ}{\stackrel{\circ}{n}}$ & 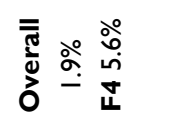 & $\stackrel{\circ}{\stackrel{\circ}{-}}$ & 1 & 1 & 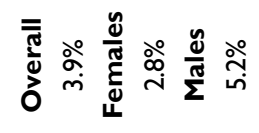 & 1 & 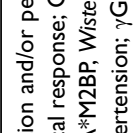 \\
\hline 1 & 1 & 1 & ঐे & 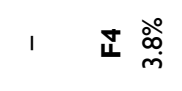 & 1 & 1 & 1 & 1 & 1 & 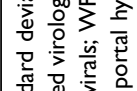 \\
\hline 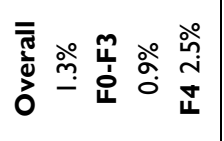 & 1 & 高 & 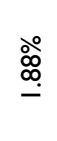 & 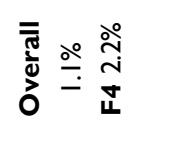 & ํํํ & 1 & 1 & 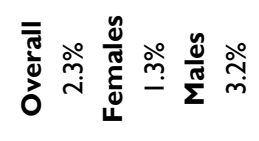 & 1 & 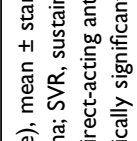 \\
\hline \multirow[t]{2}{*}{1} & 1 & 1 & $\frac{\circ 0}{\circ}$ & 1 & 1 & 1 & 1 & 1 & 1 & 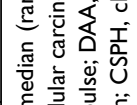 \\
\hline & 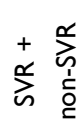 & $\stackrel{\alpha}{s}$ & 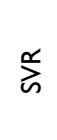 & $\stackrel{x}{\text { in }}$ & & $\stackrel{\alpha}{s}$ & & & $\stackrel{o}{s}$ & 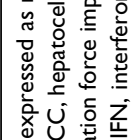 \\
\hline $\begin{array}{l}\frac{0}{0} \\
\frac{1}{2} \\
\dot{0} \\
\underline{0}\end{array}$ & 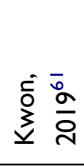 & 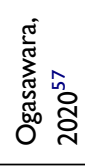 & 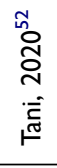 & 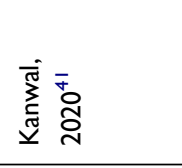 & 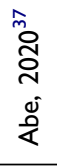 & 旅 & 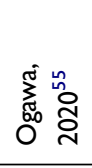 & 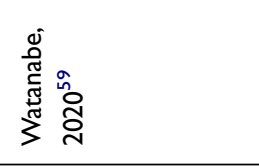 & 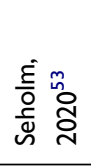 & 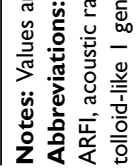 \\
\hline
\end{tabular}




\begin{tabular}{|c|c|c|c|c|c|c|c|c|c|c|c|c|c|c|c|c|}
\hline & & 1 & 1 & 1 & 1 & 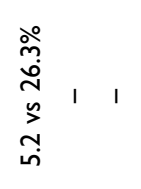 & 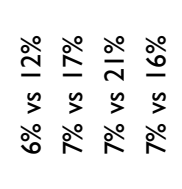 & 11 & 1 & 1 & 1 & $\begin{array}{llll}1 & 1 & 1 & 1\end{array}$ & 1 & 1 & 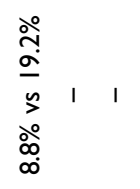 & 11 \\
\hline & & 1 & 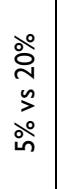 & $\begin{array}{l}\stackrel{\circ}{0} \\
\stackrel{5}{2} \\
\stackrel{\circ}{\circ}\end{array}$ & 1 & 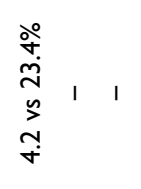 & $1 \quad 1 \quad 1$ & 11 & 1 & 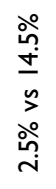 & 1 & $\begin{array}{llll}1 & 1 & 1 & 1\end{array}$ & 1 & 1 & 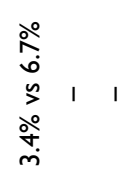 & 11 \\
\hline & & 1 & 1 & 1 & 1 & 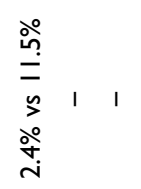 & $1 \quad 1 \quad 1$ & 11 & 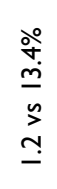 & 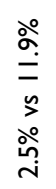 & 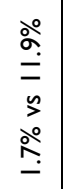 & $\begin{array}{llll}1 & 1 & 1 & 1\end{array}$ & 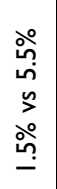 & 1 & 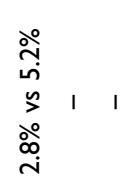 & 11 \\
\hline & & 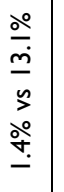 & 1 & 1 & 1 & 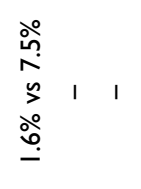 & $\begin{array}{llll}1 & 1 & 1 & 1\end{array}$ & 11 & $\begin{array}{l}\frac{0}{0} \\
0 \\
\\
\stackrel{-}{1}\end{array}$ & 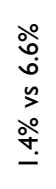 & 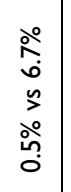 & $\begin{array}{llll}1 & 1 & 1 & 1\end{array}$ & $\begin{array}{l}\stackrel{\circ}{0} \\
\dot{m} \\
\stackrel{5}{ } \\
\stackrel{\circ}{\circ} \\
\stackrel{m}{\longrightarrow}\end{array}$ & 1 & 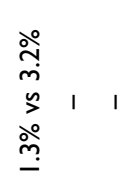 & 11 \\
\hline & & 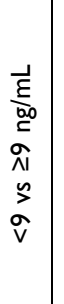 & 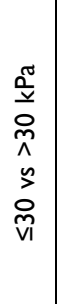 & $\begin{array}{l}a \\
\hat{n} \\
a \\
a i\end{array}$ & 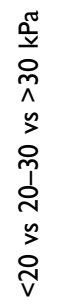 & 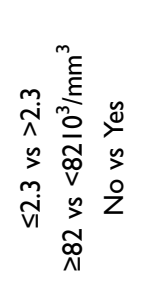 & 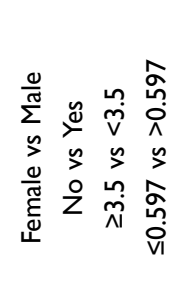 & 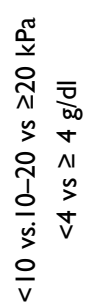 & 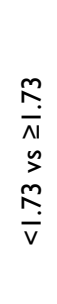 & 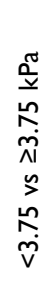 & 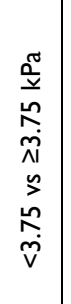 & 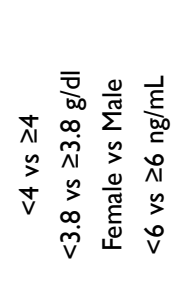 & 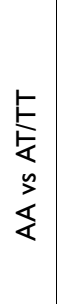 & $\begin{array}{l}\frac{\pi}{2} \\
\frac{2}{n} \\
\frac{N}{N 1} \\
5 \\
\frac{n}{n} \\
\frac{1}{v}\end{array}$ & 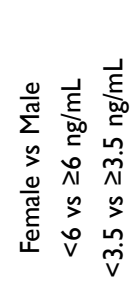 & 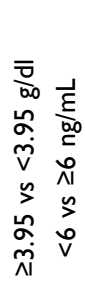 \\
\hline & & 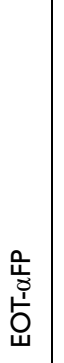 & 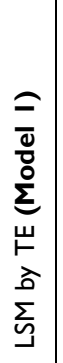 & 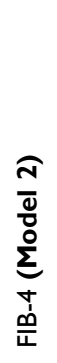 & 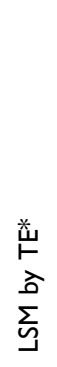 & 爱恶巻 & 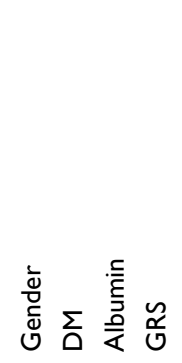 & 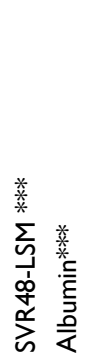 & 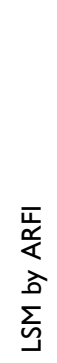 & 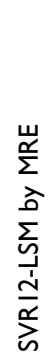 & 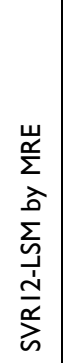 & 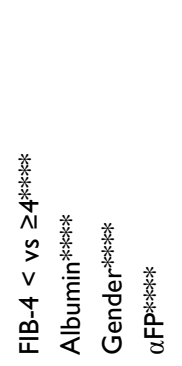 & $\bar{\exists}$ & 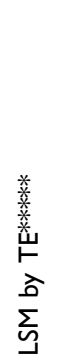 & 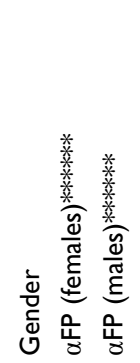 & 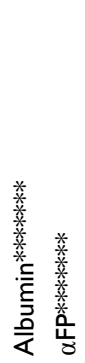 \\
\hline & & 过 & 芒 & & 过 & 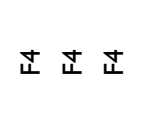 & む゙ & 号 & $\stackrel{u}{U}$ & Ư⿱ & $\stackrel{u}{\text { U }}$ & U⿱宀 & $\stackrel{u}{\underline{U}}$ & $\stackrel{u}{\mathrm{U}}$ & 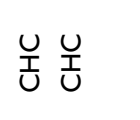 & 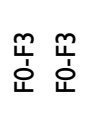 \\
\hline & & 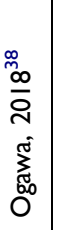 & 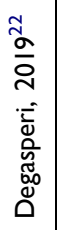 & & 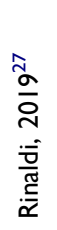 & 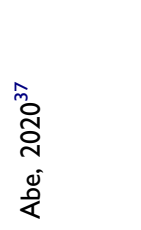 & 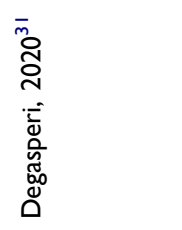 & 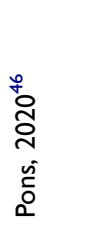 & 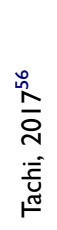 & 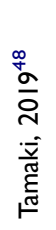 & 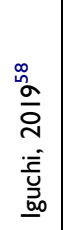 & 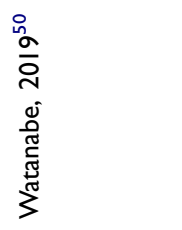 & 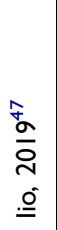 & 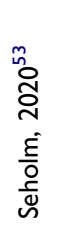 & 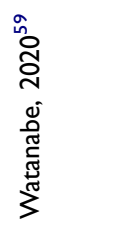 & 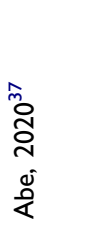 \\
\hline
\end{tabular}




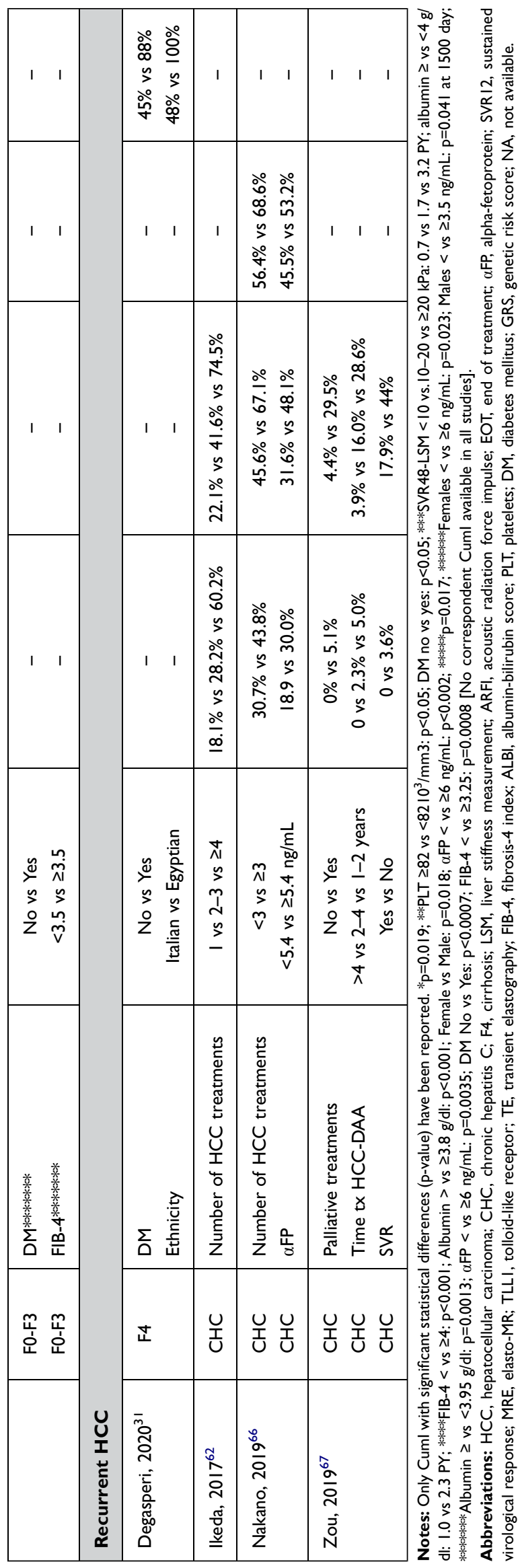

Cumulative incidences (CumI) for each study are reported in Table 3.

\section{Severity of Liver Disease}

In the setting of cirrhosis, severity of liver disease was identified among the most important predictors of de-novo $\mathrm{HCC}$, and was assessed either through non-invasive tests for fibrosis staging or clinically.

\section{Liver Stiffness Measurement (LSM)}

Several studies reported an association between de-novo HCC and LSM, mainly assessed by transient elastography (TE). LSM was correlated with HCC occurrence either when analysed at a single time-point [mostly pre-DAA (baseline)], or as a dynamic variable, by evaluating changes in LSM values between pre- and post-treatment. Thresholds able to discriminate patients at different risk of HCC development varied according to studies (Table 6). For example, Degasperi et al found that the 3-year probability of HCC significantly increased in cirrhotics with baseline LSM values $>30 \mathrm{kPa}$, while Rinaldi et al used the $20 \mathrm{kPa}$ and $30 \mathrm{kPa}$ thresholds ${ }^{22,27}$ (Table 6). In addition, Ravaioli et al reported an increased risk of de-novo HCC in patients with a $<30 \%$ decrease in LSM values, between baseline and the end of treatment (EOT) ${ }^{25}$

In F3-F4 patients, one European study reported that high baseline TE values as well as changes in LSM ( $\triangle \mathrm{LSM})$ one-year after EOT were associated with an increased risk of de-novo HCC. Pre-treatment LSM $>17.3 \mathrm{kPa}$ and $\Delta \mathrm{LSM}>25.5 \%$ were finally included in a predictive model (see below) ${ }^{45}$ (Table 7). These results were not confirmed by Pons et al, reporting that the risk of de-novo HCC in CACLD patients was independent of LSM improvement, either when using the $30 \%$ or $20 \%$ decline cut-offs. Conversely, the risk of de-novo HCC was increased by LSM values $>10 \mathrm{kPa}$ one-year after EOT. ${ }^{46}$

\section{Serological Non-Invasive Tests (NITs)}

Among NITs, Fibrosis-4 Index (FIB-4) was the most used to assess fibrosis severity. Baseline FIB-4 emerged as an independent risk factor for de-novo $\mathrm{HCC}$ in some studies analysing cirrhotic patients, ${ }^{22,26,40}$ although different cutoffs were identified (Table 6). Degasperi et al reported a significantly higher 3-year de-novo $\mathrm{HCC}$ incidence in patients with baseline FIB- $4>9$, while Ide et al identified the alternative 4.6 cut-off, that was therefore incorporated in a composite predictive score (see below) ${ }^{40}$ (Table 7). In F3-F4 patients, Alonso Lopez et al found that both 


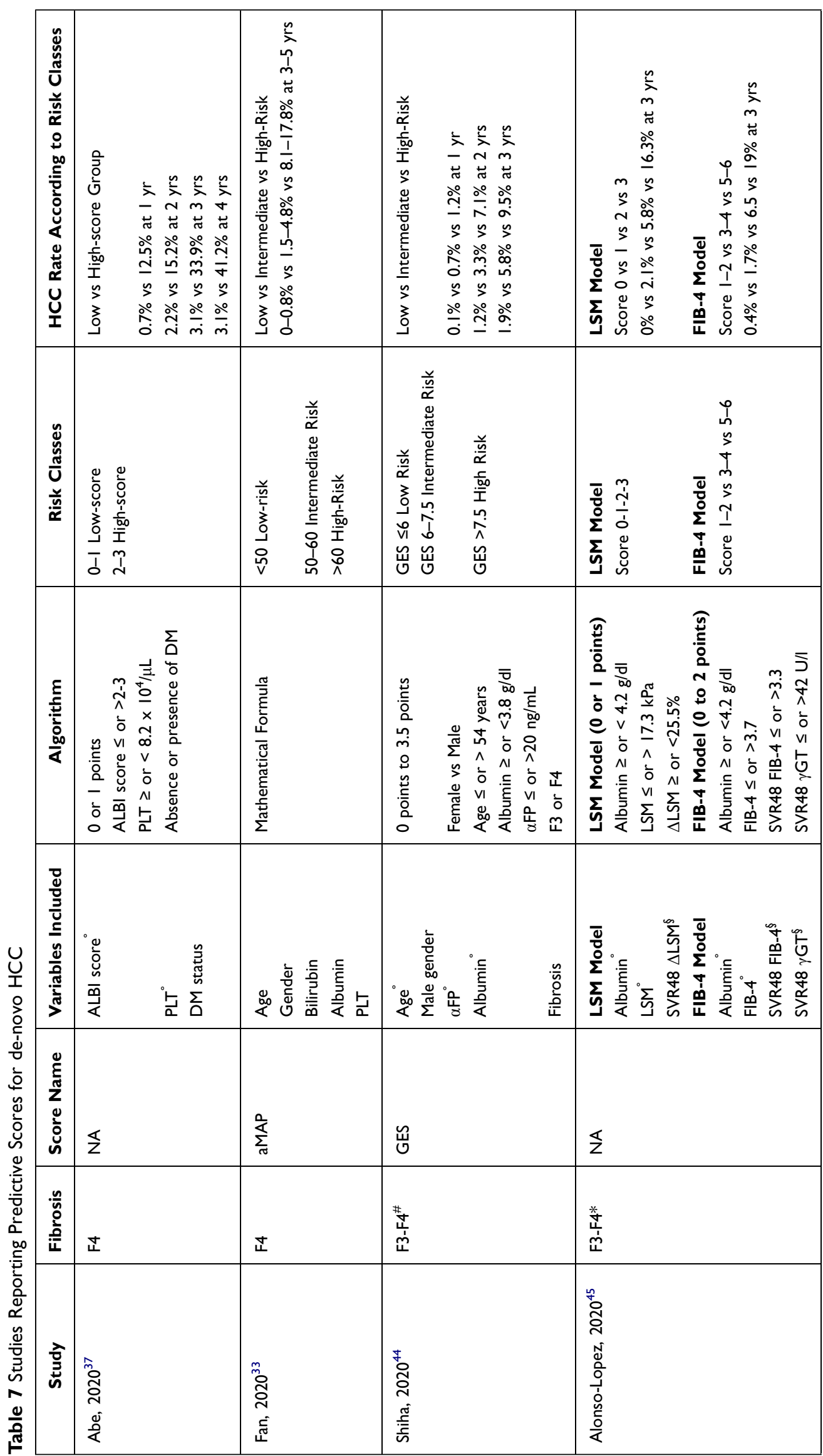




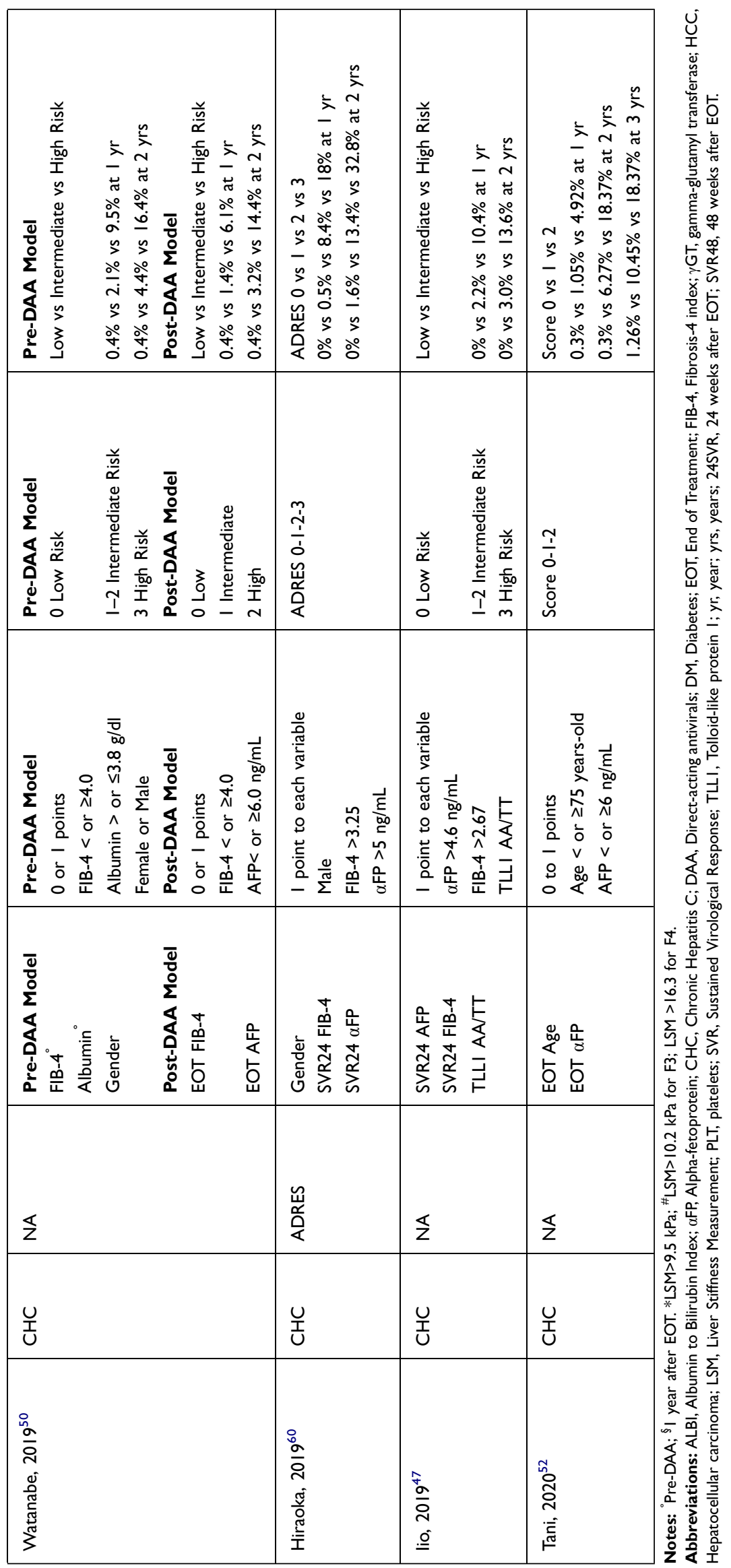


baseline FIB- $4>3.7$ and FIB- $4>3.3$ one year after treatment were associated with de-novo $\mathrm{HCC}^{45}$ (Table 7). Other NITs had been investigated as predictors of HCC in several studies: the albumin-bilirubin (ALBI) score grade $2-3^{29,37,42}$ and AST to platelet (PLT) ratio index $($ APRI $)>2.5^{11}$ emerged as independent risk factors for de-novo $\mathrm{HCC}$ in patients with cirrhosis and advanced fibrosis, respectively.

\section{Portal Hypertension and Surrogates of Advanced Liver Disease}

The risk of de-novo HCC was also increased in cirrhotic patients with clinical features of more advanced liver disease, irrespective of LSM and/or NIT values. The presence of portal hypertension $(\mathrm{PH})$ was an independent predictor of $\mathrm{HCC}$ in several studies, although definition of PH was heterogeneous. Ogawa et al defined PH by either LSM values $(\geq 20 \mathrm{kPa}$ ) or hepatic venous pressure gradient (HVPG; $>10 \mathrm{mmHg}$ ), or by imaging. ${ }^{38}$ Thus, among indirect markers of $\mathrm{PH}$ were both biochemical tests and clinical features. For example, albumin ${ }^{20,31,37,44,45}$ and PLT $^{20,21,27,29}$ were independently associated with HCC occurrence, either as single predictors or when included in predictive scores (Tables 6 and 7). The presence of esophageal varices $(\mathrm{EV})^{28}$ or ascites was associated with an increased the risk of de-novo HCC. ${ }^{32}$

Clinical scores incorporating these parameters, such as Child-Pugh-Turcotte (CPT) ${ }^{25,36}$ and Model for End Stage Liver Disease (MELD) ${ }^{41}$ or ALBI scores ${ }^{29,37,42}$ were independently associated with de-novo HCC in cirrhotics (Table 7). Some studies provided different CumI of denovo HCC according to the combination of one or more variables associated with portal hypertension (see below).

\section{Patient-Related Factors}

Several patients' features, either modifiable or not modifiable, have been shown to increase the risk of de-novo HCC after the achievement of an SVR. Age was independently associated with HCC occurrence in most studies ${ }^{21,27,28,40-}$ 42,44 as well as male gender $22,26,31,36,40,44$ (Tables 3 and 5). In US cohorts, a role of non-African American ethnicity has been suggested, ${ }^{34,41}$ although this association deserves further confirmation. Among comorbidities, diabetes mellitus (DM) has been associated with an increased risk of HCC in several cohorts; ${ }^{22,26,31,36,37}$ Abe et al incorporated DM status in a multivariable HCC risk score (see below) (Table 7). Other factors such as alcohol consumption, ${ }^{21,41}$ and viral co-infections ${ }^{11}$ are likely to influence post-SVR
HCC risk, although these patients were systematically excluded from most clinical trials.

\section{Genetic Predictors}

A single-center study conducted in a large cohort of DAAtreated cirrhotic patients found that a genetic risk score combining 4 single nucleotide polymorphisms (SNPs) [PNPLA3, TM6SF2, MBOAT7 and GCKR] was an independent predictor of de-novo $\mathrm{HCC}$, together with other clinical predictors (DM, male gender, albumin values) ${ }^{31}$ (Tables 3 and 6). The same authors found that the tolloidlike 1 (TLL1) gene, which had been previously associated with HCC occurrence in Japanese CHC patients, ${ }^{47}$ did not predict de-novo HCC in 348 European cirrhotics. ${ }^{26}$

\section{Virus-Related Factors}

Two studies, only, reported that HCV genotype might influence the risk of HCC during follow-up. Nahon et al found that $\mathrm{HCV}-1$ patients were at increased risk of $\mathrm{HCC}$ development, ${ }^{21}$ whilst genotype 3 was independently associated with de-novo $\mathrm{HCC}$ in a large retrospective study from $\mathrm{US}^{41}$ (Table 3 ).

\section{Alpha-Fetoprotein ( $\alpha \mathrm{FP})$}

Although not universally recommended for HCC surveillance by international guidelines due to its low sensitivity and specificity, broad application of $\alpha \mathrm{FP}$ in routine clinical practice has led many authors to investigate its potential for de-novo HCC prediction. $\alpha$ FP was independently associated with HCC occurrence, either in patients with cirrhosis ${ }^{32,38,42}$ or advanced fibrosis. ${ }^{44}$ Some studies evaluated the predictive ability of $\alpha \mathrm{FP}$ assessed at baseline, ${ }^{32,42,44}$ while others analysed the EOT time-point. ${ }^{38}$ Most studies tried to identify a predictive $\alpha$ FP cut-off: overall, the proposed cut-offs resulted always higher than the reference standard $7 \mathrm{ng} / \mathrm{mL}$ (ie, $>9$ or $\geq 10 \mathrm{ng} / \mathrm{mL},>20 \mathrm{mg} / \mathrm{mL})^{38,42,44}$ (Tables 3 and 6).

\section{Undefined Nodules}

Sangiovanni et al found that the presence of undefined/ non-malignant nodules at baseline was an independent predictor of HCC occurrence in cirrhotic patients. ${ }^{32}$ Partially in line with this finding is what reported by Tamaki et al, as they found that Li-Rads $3 / 4$ nodules were independently associated with $\mathrm{HCC}$ occurrence in CHC patients $(6.9 \%$ cirrhotics $) .{ }^{48}$ To avoid biases related to inclusion of patients carrying nodules at risk of HCC transformation, presence of undefined nodules was declared to be an exclusion criterion in some studies $^{10,22,26,31,36}$ (Table 3). 


\section{Lack of a Sustained Virological Response}

Some studies including large cohorts of treated patients did not allow separate analysis of those achieving an SVR, thus leading to include non-SVR among potential predictors of de-novo HCC. Although the statistical power when analysing the influence of non-SVR status on HCC risk, some authors reported that the lack of an SVR was asso-

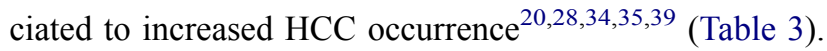

\section{Combined Predictors and HCC Risk Scores}

The risk of de-novo HCC increased when two or more independent predictors identified at multivariable analysis were combined. Not surprisingly, in all cases HCC cumulative incidences (CumI) proportionally increased according to the number of risk factors considered. ${ }^{11,20,22,28,46}$ These studies mostly included parameters associated with liver disease severity (LSM, APRI, CPT score, PLT, albumin), DM and SVR status. Conversely, other studies evaluated composite HCC risk scores, which were based on combinations of multiple variables, to stratify patients into different HCC-risk classes. Four studies focused only on patients with advanced fibrosis or cirrhosis, by proposing a combination of patient-related (age, gender, presence of DM) and biochemical variables (albumin, $\gamma \mathrm{GT}, \mathrm{PLT}, \alpha \mathrm{FP})$ together with data related to liver disease severity. ${ }^{33,37,44,45}$ The aMAP score failed in predicting de-novo $\mathrm{HCC}$ in $2085 \mathrm{~F} 3-\mathrm{F} 4$ patients with $\mathrm{HCV}-4,{ }^{49}$ and, similarly, GES score performance was suboptimal in a Caucasian cohort. ${ }^{30}$ Cumulative incidences of de-novo $\mathrm{HCC}$ according to different risk classes are reported in Table 7.

\section{Studies Enrolling Patients with Chronic Hepatitis C (Any Fibrosis Stage)}

Twenty-three studies reported data on HCC occurrence in CHC patients with any stage of liver fibrosis (Table 3). Almost all these studies included cirrhotic patients; rates of cirrhotics ranged between $6 \%$ and $73 \%$, although some authors did not provide this information. Most studies included only patients with an SVR, whilst data could be extrapolated from three studies. ${ }^{34,42,50}$ In studies including non-SVR patients, treatment failure accounted for 3.0 $6.7 \%$ of DAA treatment responses (Table 4).

Overall, $0.9 \%$ to $6.9 \%$ of CHC patients developed denovo HCC during follow-up, although only few authors reported the prevalence of cirrhosis in $\mathrm{CHC}$ cohorts. When reported, rates of cirrhosis were between $38 \%$ and $100 \%$ in
CHC patients developing $\mathrm{HCC}^{35,39,40,51-53}$ (Table 4), and overall CumI of de-novo HCC were lower than that reported in cirrhotic cohorts, at each time-point (Tables 2 and 4).

\section{Severity of Liver Disease}

Due to the inclusion of cirrhotic patients in CHC cohorts, liver disease severity was independently associated with HCC occurrence in most studies. Only 5 studies were able to identify $\mathrm{HCC}$ predictors in non-cirrhotic F0-F3 patients, ${ }^{34,37,38,40,42}$ and three of them included indirect markers of fibrosis, either biochemical tests or NITs (see below) (Table 5).

\section{Cirrhosis and Advanced Fibrosis}

Whatever defined, cirrhosis and advanced fibrosis were independently associated with HCC development in several studies. ${ }^{34,38,39,54,55}$ In these studies, cirrhosis was differently defined (Table 1), and ranged between $18 \%$ and $56 \%$ of the overall population. Particularly, the CHC cohort described by Pinero et al included 399 (29\%) patients with clinically significant portal hypertension (CSPH) (Table 4).

\section{Liver Stiffness Measurement}

Baseline LSM obtained by Acoustic Radiation Force Impulse (ARFI) or TE, was associated with an increased risk of post-treatment HCC (Table 5) in three studies analysing SVR patients. ${ }^{53,56,57}$ Tachi et al identified the $1.73 \mathrm{~m} / \mathrm{s}$ threshold as the optimal cut-off to stratify CHC patients according to their de-novo HCC risk $^{56}$ (Table 5). In the other two studies, baseline LSM $\geq 20 \mathrm{kPa}$ and $\geq 17.5$ $\mathrm{kPa}$ were associated with HCC occurrence in 398 and 773 CHC patients from Japan and Denmark, respectively. In both studies, the prevalence of cirrhosis was not reported.53,57 Ogasawara et al reported that also SVR24-LSM (ie, LSM performed 24 weeks after EOT) $\geq 10 \mathrm{kPa}$ was independently associated with de-novo HCC. ${ }^{57}$ This finding was in line with two other Japanese studies, identifying in LSM $\geq 3.75 \mathrm{kPa}$ obtained through Magnetic Resonance Elastography (MRE) 12 weeks after treatment completion (SVR12-MRE) an independent predictor of HCC occurrence. $^{48,58}$

\section{Serological Non-Invasive Tests}

Most studies reported that either baseline or post-treatment FIB-4 values were associated with the risk of de-novo $\mathrm{HCC}$ in CHC patients. In 5 studies, baseline FIB-4 was 
reported to be an independent risk factor for $\mathrm{HCC}$ occurrence. ${ }^{34,40,47,50,59}$ Risk thresholds varied according to each study: Kanwal et al used the standard 3.25 cutoff, ${ }^{34}$ whereas lio et al used the 2.67 cut-off. ${ }^{47}$ In the study by Watanabe et al, baseline FIB-4 predicted HCC in females, only. ${ }^{59}$ In addition, three Japanese studies reported that post-SVR FIB-4 (at EOT and at SVR12) and changes in FIB-4 independently predicted de-novo HCC. ${ }^{50,55,60}$ Among investigated serological biomarkers of fibrosis was Wisteria floribunda agglutinin positive Mac-2 (WFA*M2BP), which was tested in the study by Nagata et al, reporting that WFA*M2BP assessed 24 weeks after EOT independently predicted de-novo $\mathrm{HCC}^{55}$ (Tables 5 and 6).

\section{Biochemical Surrogates of Advanced Liver Disease}

Albumin and PLT were independently associated with HCC occurrence in two studies reporting data on F0-F3 patients. ${ }^{37,38}$ Also, Watanabe et al found that low pretreatment albumin values $(<3.8 \mathrm{~g} / \mathrm{dl})$ increased the risk of $\mathrm{HCC}^{49}$ (Tables 5-7).

\section{Patient-Related Factors}

Male gender and age independently predicted HCC occurrence also in CHC cohorts. Male gender was associated with de-novo $\mathrm{HCC}$ in $5^{40,50,55,59,60}$ studies, and age in $7^{40,41,48,52,53,55,58}$ (Table 5). Although age was analysed as a continuous variable in multivariate analysis, different cut-offs were associated with increased risks of HCC $(>60,>62,>75$ years). Race still emerged as independent predictor of de-novo HCC in the large US cohorts, ${ }^{34,41}$ but was not confirmed by other studies. Co-morbidities influenced HCC development also in CHC cohorts: Kanwal et al reported that the presence of DM was independently associated with HCC occurrence, ${ }^{34}$ whereas other studies found that altered $\gamma \mathrm{GT}$ and ALT values predicted post-SVR HCC, ${ }^{40,55}$ likely mirroring the presence of underlying metabolic disorders (Tables 5 and 6).

\section{Genetic Predictors}

In $\mathrm{CHC}$ patients, two genetic factors were independently associated with HCC occurrence. Nagata et al found that IL28B rs8099917 polymorphism (non-TT) was associated with an increased risk of HCC in a large cohort of 752 patients followed-up for 1.8 years. ${ }^{54}$ Conversely, the Japanese study by Iio et al reported that patients carrying the TLL1 rs17047200 AT/TT genotypes had significantly higher CumI of HCC, although $\mathrm{T}$ allele was associated with lower PLT and higher FIB-4 values. ${ }^{47}$ In 348 F0-F3 patients from Italy, TLL1 genotype did influence HCC risk $^{26}$ (Table 5).

\section{Alpha-Fetoprotein}

Baseline $\alpha \mathrm{FP}$ was independently associated with de-novo HCC in 4 studies, which however identified different cutoffs: $>4.6 \mathrm{ng} / \mathrm{mL} \geq 8 \mathrm{ng} / \mathrm{mL}$ and $\geq 10 \mathrm{ng} / \mathrm{mL}^{42,47,55,57}$ (Table 5). In addition, some authors investigated the predictive values of post-treatment $\alpha \mathrm{FP}$ (Table 5): values at both EOT, ${ }^{50-52,59}$ and SVR12 $2^{48,58,60}$ time-points were associated with de-novo HCC. At SVR12, the following cut-offs were identified: $>5 \mathrm{ng} / \mathrm{mL},>6.5 \mathrm{ng} / \mathrm{mL}, \geq 6 \mathrm{ng} /$ $\mathrm{mL}$. Interestingly, Watanabe et al proposed two different cut-offs (ROC analysis) for post-treatment $\alpha \mathrm{FP}$ according to patient gender: $>6.0 \mathrm{ng} / \mathrm{mL}$ in females and $>3.5 \mathrm{ng} / \mathrm{mL}$ in males, respectively ${ }^{59}$ (Tables 5 and 6).

\section{Virus-Related Factors}

The only study reporting a role of virus-related factors is the one by Kanwal et al, finding an association between HCV genotype 3 and de-novo HCC. ${ }^{41}$

\section{Combined Scores}

In the setting of $\mathrm{CHC}$ patients, 4 studies developed scores based on multiple variables to predict de-novo HCC, mostly assessed at EOT or SVR time-points. Hiraoka et al proposed the ADRES score, based on the combination of gender, FIB-4 and $\alpha$ FP assessed at SVR24, while Tani et al incorporated EOT- $\alpha$ FP $(>6 \mathrm{ng} / \mathrm{mL})$ and age $(>75$ years $^{52,60}$ (Table 7). Iio et al combined SVR24- $\alpha$ FP and FIB-4 with the TLL1 genotype, ${ }^{47}$ while Watanabe et al proposed two different models, either pre-DAA (including FIB-4, albumin and gender) or post-DAA (incorporating EOT- FIB-4 and $\alpha$ FP values) ${ }^{50}$ (Table 7).

\section{Predictors of Recurrent HCC}

The risk of HCC following antiviral treatment was strongly influenced by previous HCC history. Not only rates of recurrent $\mathrm{HCC}$ were significantly higher than those of de-novo HCC (Tables 2, 4, 5 and 8), but previous HCC history was the strongest predictor of HCC development in cohorts analysing cumulative data from patients with and without pre-DAA liver cancer. Rates of HCC recurrence following DAA were $\operatorname{similar}^{18,19,23,61}$ or even lower $^{62}$ than those reported in untreated patients, and most authors reported that oral antivirals did not enhance the risk of recurrence. ${ }^{12,15,18,19,23,61}$ 


\begin{tabular}{|c|c|c|c|c|c|c|c|c|c|c|c|c|}
\hline 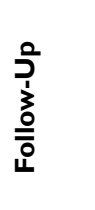 & & $\begin{array}{l}3 \\
3 \\
4 \\
4\end{array}$ & 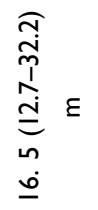 & 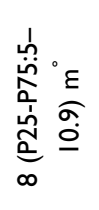 & $\S$ & $\begin{array}{l}{ }^{o} \varepsilon \\
\sigma \\
\frac{\sigma}{1} \\
m \\
0 \\
\infty \\
\infty\end{array}$ & 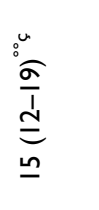 & 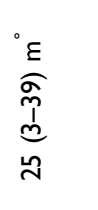 & $\S$ & $\stackrel{u}{\circ} \geq$ & 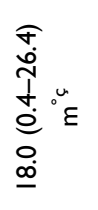 & 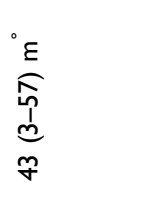 \\
\hline 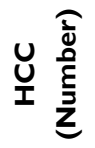 & & 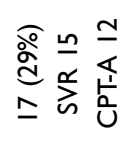 & $\begin{array}{l}\stackrel{\circ}{\stackrel{0}{5}} \\
-\end{array}$ & 官 & 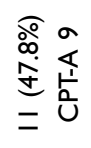 & $\begin{array}{l}\text { @े } \\
\text { O্ं } \\
\text { ì }\end{array}$ & $\begin{array}{l}\text { ळ. } \\
\stackrel{0}{0} \\
\stackrel{0}{n} \\
\end{array}$ & 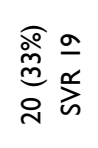 & 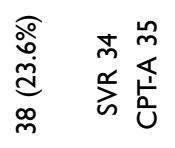 & 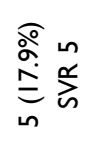 & $\begin{array}{l}\text { ळo } \\
0 \\
0 \\
0 \\
0 \\
0\end{array}$ & 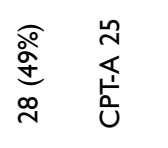 \\
\hline$\sum_{n}^{\infty}$ & & 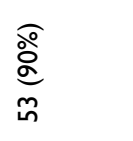 & $\begin{array}{l}\text { o̊을 } \\
\text { m }\end{array}$ & तิ & 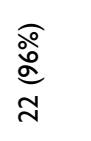 & 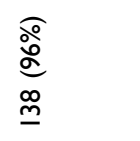 & 乏 & $\begin{array}{l}\text { 产 } \\
\vdots\end{array}$ & 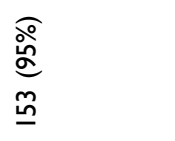 & 㐫 & $\S$ & 足 \\
\hline ปั & & 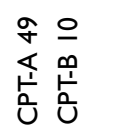 & 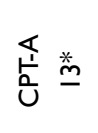 & 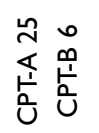 & 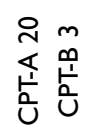 & 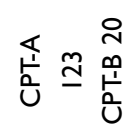 & $\S$ & 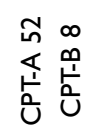 & 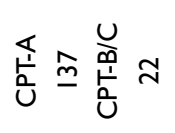 & $\S$ & 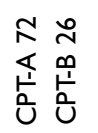 & 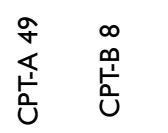 \\
\hline 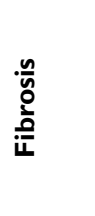 & & 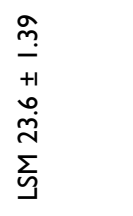 & $\S$ & $\S$ & $\S$ & $\S$ & $\S$ & 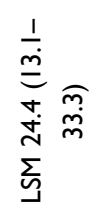 & 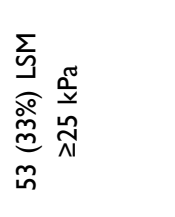 & $\S$ & $\S$ & 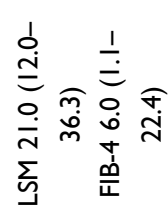 \\
\hline 获 & & 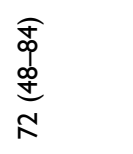 & $\begin{array}{l}\frac{0}{+1} \\
\frac{1}{6}\end{array}$ & $\begin{array}{l}\infty \\
+1 \\
1 \\
n\end{array}$ & $\begin{array}{l}\stackrel{5}{\infty} \\
\frac{1}{n} \\
\infty \\
\infty \\
\stackrel{n}{n}\end{array}$ & $\begin{array}{l}a \\
+1 \\
0\end{array}$ & ¿ & 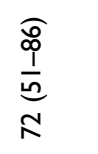 & 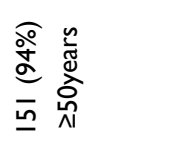 & $\S$ & $\begin{array}{l}\widehat{o} \\
\infty \\
1 \\
\hat{y} \\
\frac{1}{1}\end{array}$ & 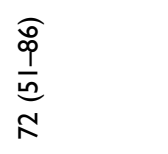 \\
\hline$\frac{\tilde{y}}{\frac{\pi}{\pi}}$ & & \& $\stackrel{\circ}{\stackrel{\circ}{0}}$ & 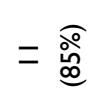 & 유 앙 & 요 $\frac{\widehat{\circ}}{\infty}$ & œ & ¿ & î స్ర్రి & $\equiv \stackrel{\circ}{\circ}$ & 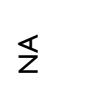 & $8 \frac{\sqrt[ఠ]{9}}{\sqrt{0}}$ & 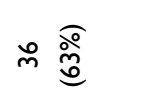 \\
\hline 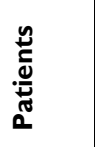 & & 官 & 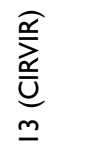 & $\bar{m}$ & $\stackrel{\sim}{\sim}$ & $\stackrel{m}{\underline{I}}$ & $\underline{\underline{ }}$ & 요 & $\underline{\overline{0}}$ & $\stackrel{\infty}{\sim}$ & $\stackrel{\infty}{\circ}$ & ถิ \\
\hline 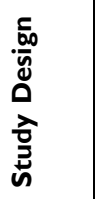 & & 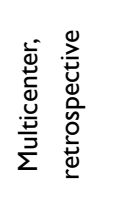 & 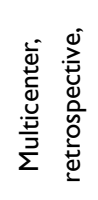 & 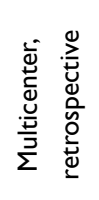 & 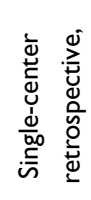 & 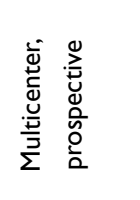 & 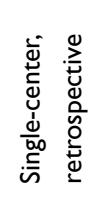 & 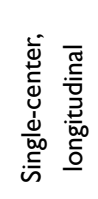 & 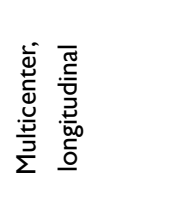 & & 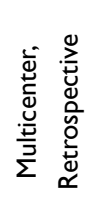 & 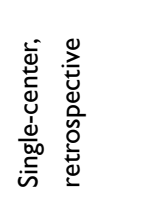 \\
\hline 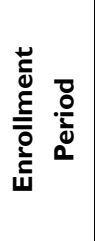 & & $\begin{array}{l}\frac{n}{2} \\
\text { N } \\
\text { त्र } \\
\text { In }\end{array}$ & 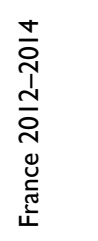 & 츨 & 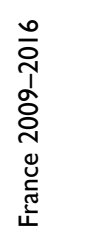 & 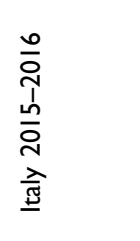 & 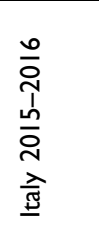 & 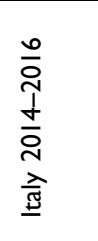 & $\begin{array}{l}\frac{n}{0} \\
\text { 조 } \\
\text { 조 }\end{array}$ & 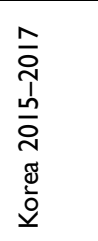 & 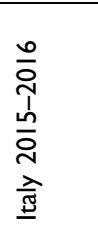 & 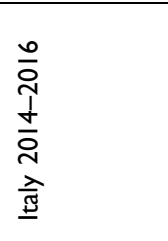 \\
\hline 高 & 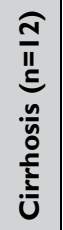 & 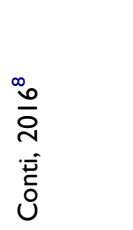 & 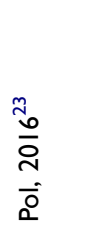 & 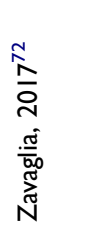 & 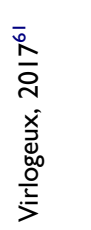 & 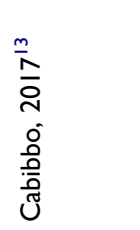 & 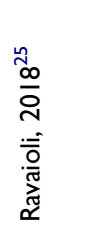 & 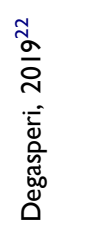 & 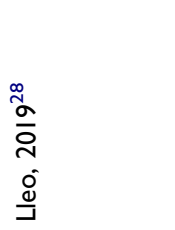 & 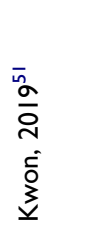 & 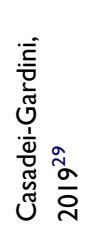 & 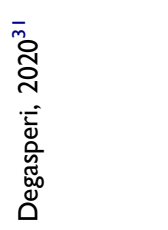 \\
\hline
\end{tabular}




\begin{tabular}{|c|c|c|c|c|c|c|c|c|c|c|}
\hline 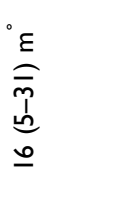 & & $\begin{array}{l}{ }^{\circ} \varepsilon \\
\frac{\varepsilon}{0} \\
+ \\
+1 \\
+ \\
0 \\
0 \\
i n\end{array}$ & $\begin{array}{l}{ }^{\circ} \varepsilon \\
\stackrel{o}{O} \\
\vdots \\
\simeq\end{array}$ & $\begin{array}{l}\stackrel{\circ}{E} \\
\text { กิ }\end{array}$ & $\begin{array}{l}\stackrel{\circ}{8} \varepsilon \\
\stackrel{\circ}{\circ}\end{array}$ & 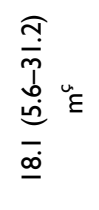 & 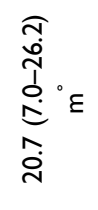 & $\vec{m}$ & $\begin{array}{l}\stackrel{u}{\varepsilon} \\
\underset{\sim}{N} \\
\stackrel{I}{=} \\
\simeq\end{array}$ & 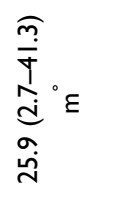 \\
\hline 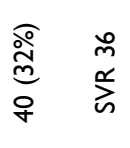 & & 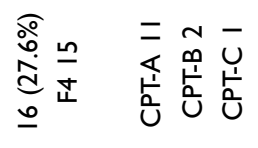 & 0 & 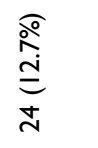 & 乏 & $\underset{\substack{\text { ò } \\
\text { I }}}{ }$ & 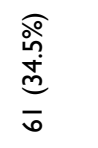 & $\begin{array}{l}\text { ঐ̊ } \\
\text { ป̃ } \\
\text { స̃ }\end{array}$ & 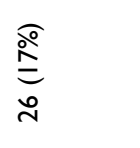 & 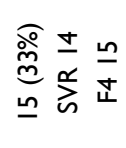 \\
\hline & & 高 & 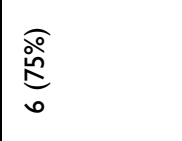 & $\stackrel{\infty}{+} \frac{\widehat{\circ}}{\sigma}$ & $\mathbb{Z}$ & $\begin{array}{l}\text { o̊ } \\
\stackrel{\circ}{\circ} \\
\text { o }\end{array}$ & 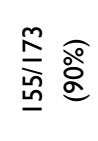 & $\S$ & 등 & 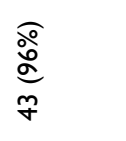 \\
\hline 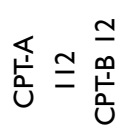 & & 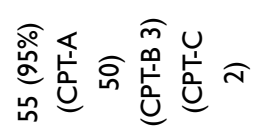 & 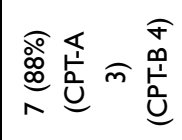 & 뜽ㅇㅇ & 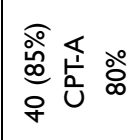 & $\mathbb{Z}$ & $\S$ & $\mathbb{Z}$ & 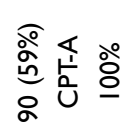 & 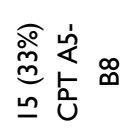 \\
\hline$\S$ & & $\S$ & $\S$ & $\S$ & $\S$ & $\S$ & $\S$ & $\S$ & $\S$ & 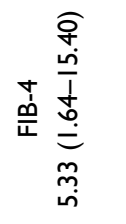 \\
\hline 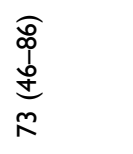 & & 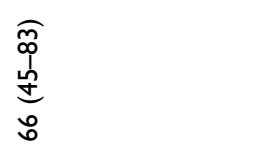 & 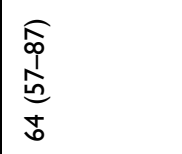 & $\begin{array}{l}a \\
+1 \\
\text { co }\end{array}$ & 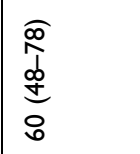 & $\mathbb{Z}$ & $\begin{array}{l}\widehat{c} \\
0 \\
o \\
0 \\
\\
r\end{array}$ & $\S$ & \begin{tabular}{l}
$\sigma$ \\
$\hat{1}$ \\
$b$ \\
$o$ \\
\multirow{1}{N}{}
\end{tabular} & $\begin{array}{l}\widehat{0} \\
0 \\
0 \\
0 \\
0 \\
\sigma\end{array}$ \\
\hline œ & & o $\underset{0}{\stackrel{\circ}{\circ}}$ & 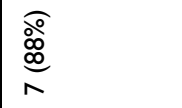 & $\hat{\underline{y}} \stackrel{\widehat{\circ}}{\stackrel{0}{c}}$ & 웅 & $\S$ & ㅇㅇㅇㅇㅇ & $\S$ & 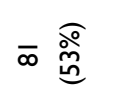 & 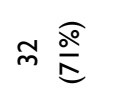 \\
\hline$\stackrel{ \pm}{I}$ & & $\stackrel{\infty}{\infty}$ & $\infty$ & 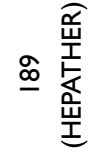 & $\hat{f}$ & ஓ్ల & $\Sigma$ & œ & 끄 & $\ddot{q}$ \\
\hline 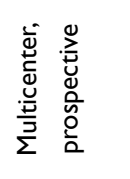 & & 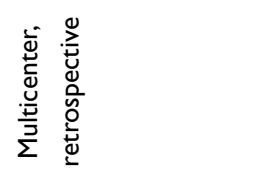 & 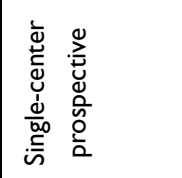 & 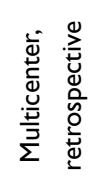 & 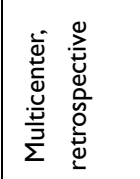 & 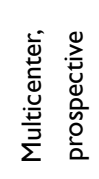 & 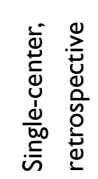 & 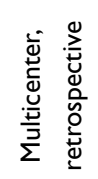 & 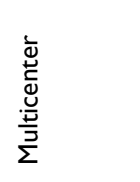 & 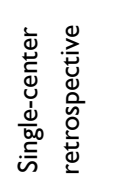 \\
\hline ब्रे & 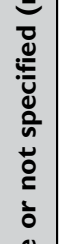 & 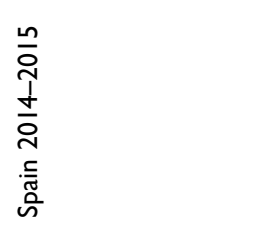 & 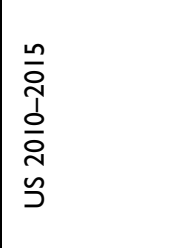 & 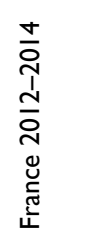 & $\begin{array}{l}\text { ळ } \\
\text { о } \\
\text { च }\end{array}$ & 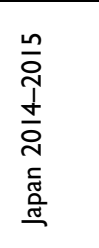 & 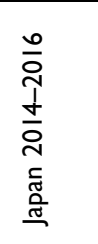 & 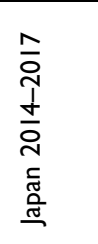 & 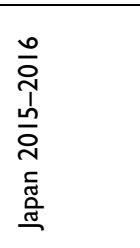 & 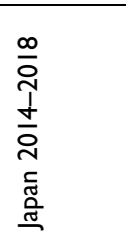 \\
\hline 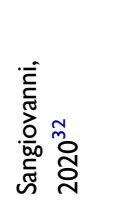 & 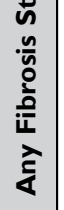 & 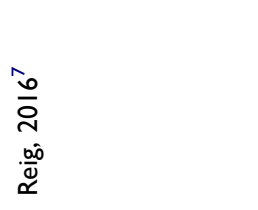 & 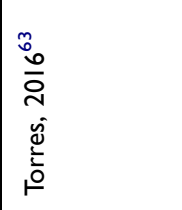 & 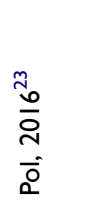 & 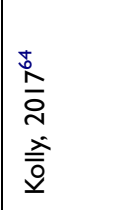 & 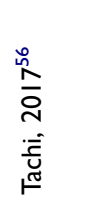 & 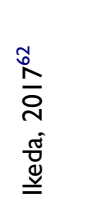 & 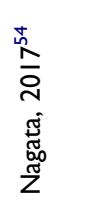 & 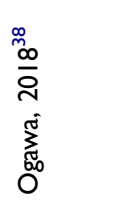 & 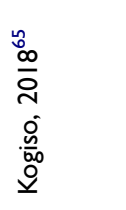 \\
\hline
\end{tabular}




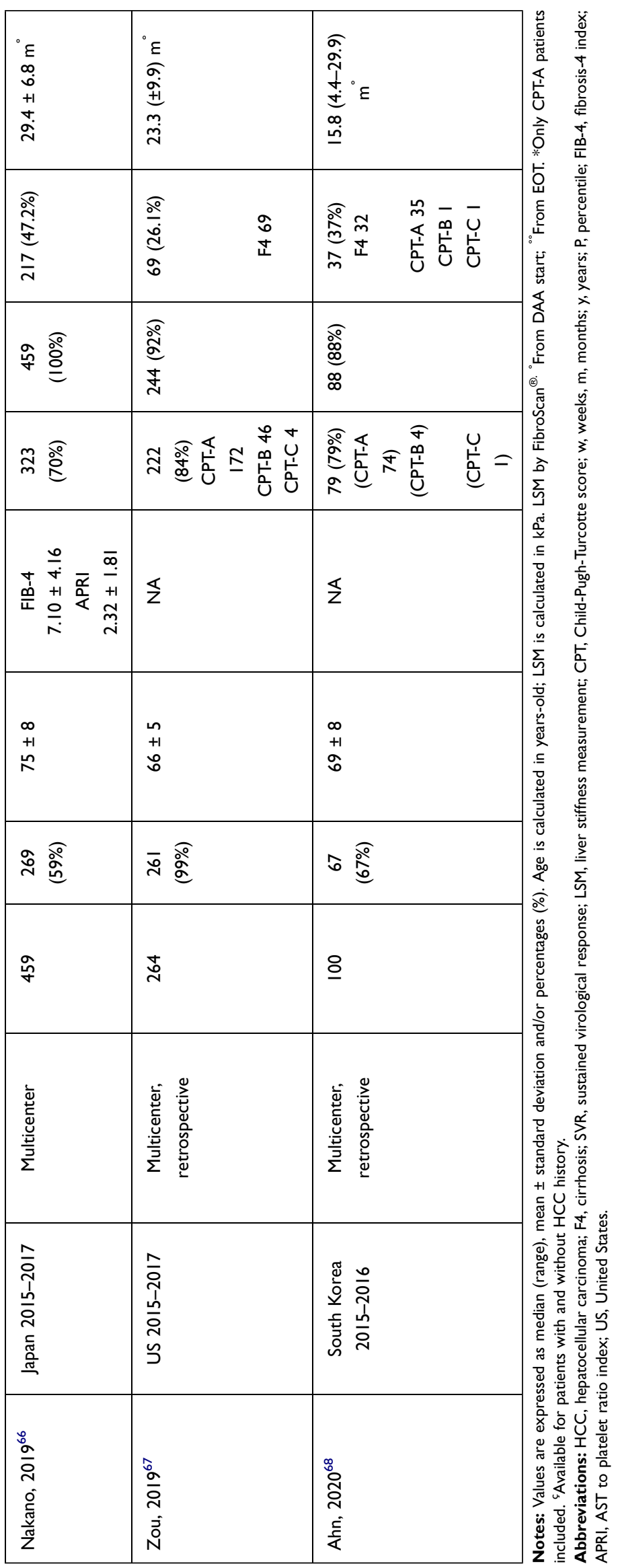


Rates of HCC recurrence ranged between $3.2 \%$ and $49 \%$, with one study only ${ }^{63}$ reporting no recurrence however among 8 patients ( 7 with cirrhosis) (Table 8 ). Almost all studies tried to identify clinical predictors of $\mathrm{HCC}$ recurrence during variable follow-up, despite the inclusion of patients with different characteristics, including tumour burden (Tables 8 and 9). In two retrospective French studies, the authors found HCC predictors different from DAA use when comparing untreated vs DAA-treated patients. ${ }^{23,61}$ Similarly, Ogawa et al were not able to identify predictive factors of HCC recurrence in 62 F0-F3 patients with an SVR, whilst other studies on $\mathrm{CHC}$ patients did not focused on the sub-group of noncirrhotics. $^{38}$ In most cases, data were obtained from cohorts including both SVR and non-SVR patients; 4 studies enrolled only cured patients, whilst this information was lacking in other 4 . When reported, rates of treatment failure ranged between $2.5 \%$ and $25 \%$ (Table 8 ). In 12 studies all patients had a diagnosis of cirrhosis, and most of them $(\mathrm{n}=9)$ included also decompensated (CPT-B) patients (Table 8). In studies enrolling $\mathrm{CHC}$ patients, rates of cirrhosis ranged between $33 \%$ and $95 \%(n=9)$ or were not reported $(n=4)$, and only few authors $(n=4)$ reported information on fibrosis stage in patients with a complete response (CR) to previous HCC who subsequently developed HCC recurrence (Table 8).

\section{Severity of Liver Disease}

Despite the inclusion of cirrhotic patients in CHC studies, only one of them was able to identify cirrhosis as an independent risk factor for HCC recurrence. ${ }^{38}$ Although not reported in most cases, expected high rates of cirrhosis in patients developing recurrent HCC might have attenuated the weight of this variable. However, further reinforcing the strength of liver disease severity as HCC predictor, some authors found that indirect markers of fibrosis were independently associated with HCC recurrence. For example, Conti et al reported that baseline LSM independently predicted HCC in 59 cirrhotics followed-up for 24 weeks, ${ }^{8}$ whereas Nagata et al found that WFA*M2BP assessed at SVR24 predicted HCC recurrence in 83 CHC patients ${ }^{54}$ (Table 9).

\section{Patient-Related Factors}

According to published studies, patients' characteristics had low impact on HCC recurrence, as only few authors found that they were independently associated with recurrent $\mathrm{HCC}$ following an SVR to DAA. However, both age ${ }^{8,64}$ and, in cirrhotic patients, comorbidities such as $\mathrm{DM}^{22,31}$ and alcohol $^{32}$ seemed to play a role in influencing HCC risk. Moreover, Degasperi et al reported that ethnicity (ie, Egyptian vs Italian) was an independent risk factor for HCC recurrence in their European cohort ${ }^{22}$ (Tables 6 and 9).

\section{Tumour Burden}

Rates of HCC recurrence were strongly influenced by tumour burden in most studies analysing either $\mathrm{F} 4$ or $\mathrm{CHC}$ cohorts (Table 9). One of the most important predictors of HCC recurrence was history of $\mathrm{HCC}$ recurrence before $\mathrm{DAA}^{8,13,32}$ together with the number of HCC treatments finally leading to $\mathrm{CR}$ achievement before anti-HCV therapies. ${ }^{62,65,66}$ In addition, time elapsing between prior HCC treatment and DAA start was significantly associated with an increased risk of recurrent HCC in several studies, ${ }^{38,64,67,68}$ where patients treated for HCC less than one year prior to DAA exhibited an increased risk of tumour recurrence. ${ }^{38,68}$ Lastly, some authors reported that also prior HCC size, ${ }^{13}$ number of nodules ${ }^{38}$ and type of HCC treatment (ie, palliative vs curative) ) $^{38,67}$ were independently associated with $\mathrm{HCC}$ recurrence, although these data were not confirmed by others ${ }^{8,22,65}$ (Table 6). However, these results should be cautiously interpreted, as they are strongly influenced by study design and patients enrollment; recently, an individual patient-data meta-analysis pooling data of 977 patients from 21 studies have further enhanced the importance of pre-DAA HCC history and tumour burden. ${ }^{19}$

\section{HCC Biomarkers}

Four studies found that higher baseline (DAA start) values of $\alpha \mathrm{FP}$ were independently associated with $\mathrm{HCC}$ recurrence. ${ }^{19,28,54,66}$ Casadei-Gardini and others found that aspartate aminotransferase to lymphocyte ratio (ALRI), which had been previously proposed for inclusion in HCC surveillance algorithms, ${ }^{69}$ independently predicted HCC recurrence in 98 cirrhotic patients (73\% CPT-A) treated with DAA 8.5 months after $\mathrm{CR}^{29}$ (Tables 6 and 9).

\section{Conclusions}

Despite the expected decrease in HCC burden, ${ }^{70}$ the widespread use of DAA to cure HCV infection will finally lead large cohorts of SVR patients to be maintained under surveillance. In fact, the number of patients requiring HCC surveillance due to pre-treatment advanced fibrosis is expected to increase over time, as a consequence of worldwide diffusion of HCV screening and treatment programs. ${ }^{71}$ Therefore, we are going to face with larger, ageing population still at risk of 


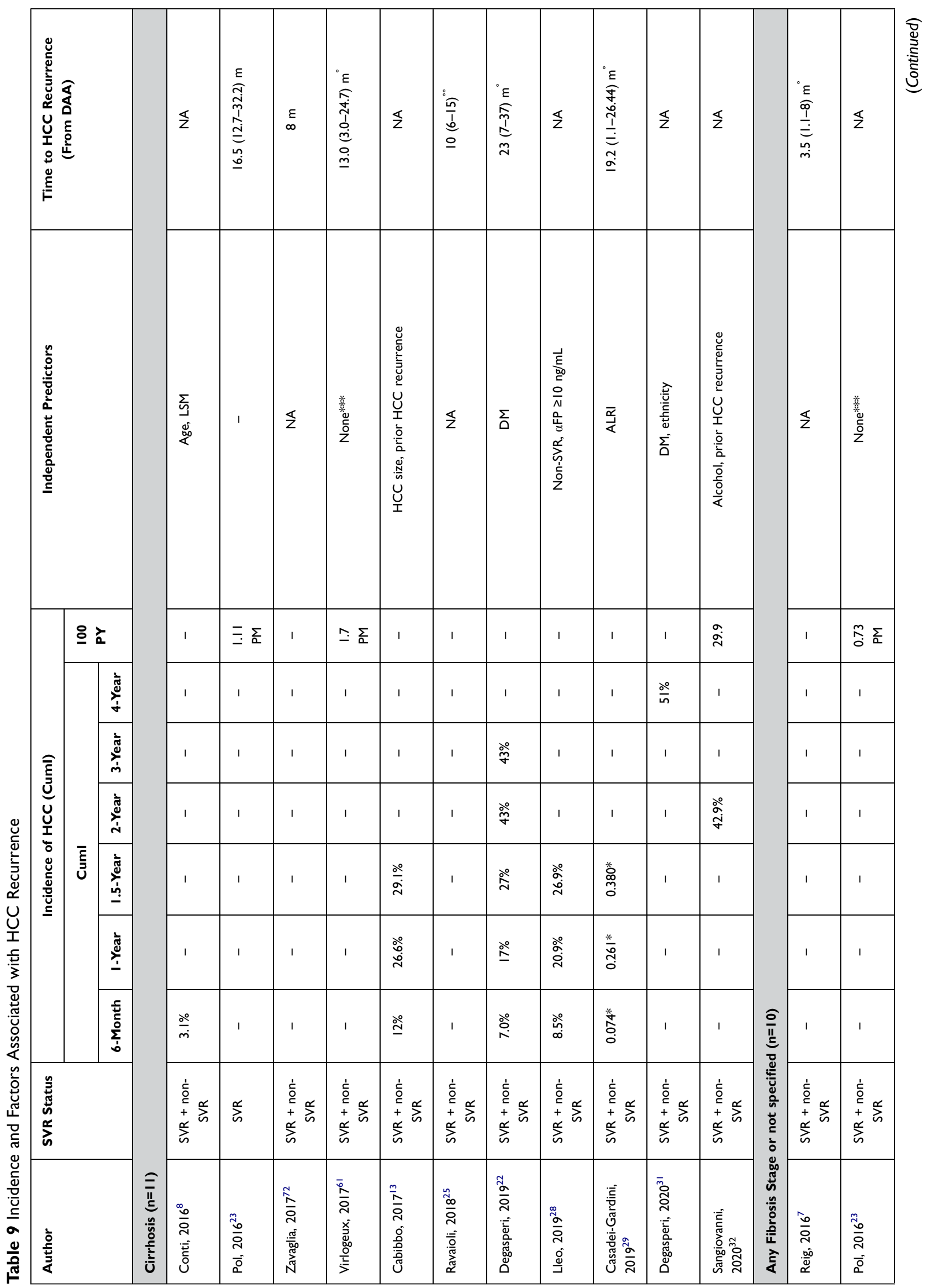




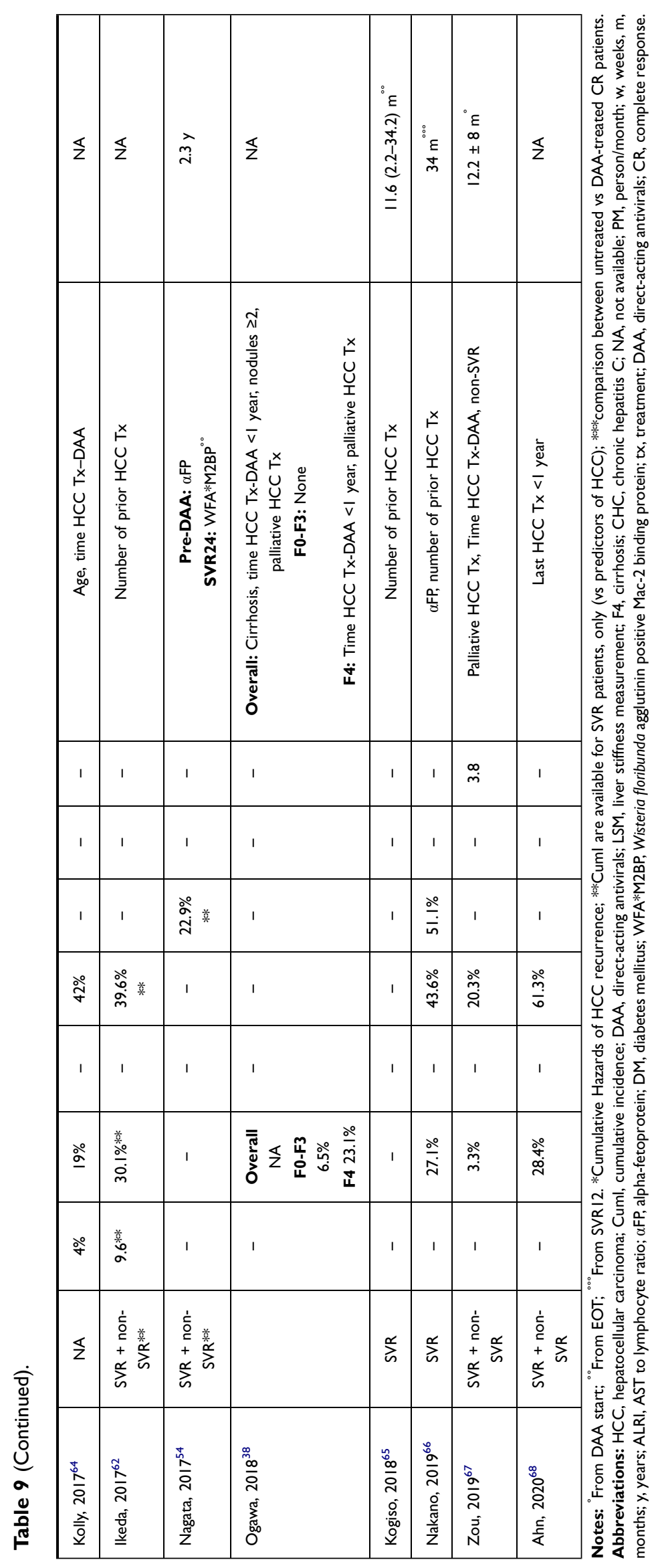


HCC, although HCC risk is lower than that reported in active $\mathrm{HCV}$ infection. As a consequence, the investigation of HCC predictors is of paramount importance in order to better optimize surveillance strategies, with the ultimate goal of personalized follow-up algorithms. While advanced fibrosis and cirrhosis represent strong predictors of HCC development, either de-novo or recurrent, literature data suggest that many co-factors may contribute to the oncogenic risk. While some of these factors are modifiable or can be potentially improved by successful antiviral treatments (fibrosis, portal hypertension), others are only partially modifiable (metabolic syndrome) or not modifiable at all (aging, HCC history). Due to the complex interactions and competing risks resulting from these variables, combination analyses or composite scores are those expected to better improve prediction capability, with all the challenges related to large-scale applicability in heterogeneous patient populations. Therefore, in most cases prospective validation in larger cohorts is still needed.

\section{Disclosure}

Roberta D'Ambrosio reports being on the advisory board for AbbVie and MSD; speaking and teaching for AbbVie, Gilead and MSD; and research support from AbbVie, Gilead and MSD, outside the submitted work. Elisabetta Degasperi reports personal fees from ABBVIE and grants, personal fees and non-financial support from GILEAD, outside the submitted work. The authors report no conflicts of interest in this work.

\section{References}

1. European Association for the Study of the Liver. EASL clinical practice guidelines: management of hepatocellular carcinoma. $J$ Hepatol. 2018;69:182-236.

2. Llovet JM, Kelley RK, Villanueva A, et al. Hepatocellular carcinoma. Nat Rev Dis Primers. 2021;7(1):6. doi:10.1038/s41572-020-00240-3

3. Van der Meer A, Veldt BJ, Feld JJ, et al. Association between sustained virological response and all-cause mortality among patients with chronic hepatitis $\mathrm{C}$ and advanced hepatic fibrosis. JAMA. 2012;308:2584-2593. doi:10.1001/jama.2012.144878

4. Morgan RL, Baack B, Smith BD, et al. Eradication of hepatitis C virus infection and the development of hepatocellular carcinoma: a metaanalysis of observational studies. Ann Intern Med. 2013;158:329-337. doi:10.7326/0003-4819-158-5-201303050-00005

5. El-Serag HB, Kanwal F, Richardson P, et al. Risk of hepatocellular carcinoma after sustained virological response in Veterans with hepatitis C infection. Hepatology. 2016;64:130-137. doi:10.1002/hep.28535

6. van der Meer AJ, Feld JJ, Hofer H, et al. Risk of cirrhosis-related complications in patients with advanced fibrosis following hepatitis $\mathrm{C}$ virus eradication. $J$ Hepatol. 2017;66:485-493. doi:10.1016/j. jhep.2016.10.017

7. Reig M, Marino Z, Perello C, et al. Unexpected high rate of early tumour recurrence in patients with $\mathrm{HCV}$-related $\mathrm{HCC}$ undergoing interferon-free therapy. $J$ Hepatol. 2016;65:719-726. doi:10.1016/j. jhep.2016.04.008
8. Conti F, Buonfiglioli F, Scuteri A, et al. Early occurrence and recurrence of hepatocellular carcinoma in HCV-related cirrhosis treated with direct-acting antivirals. $H$ Hepatol. 2016;65:727-733. doi:10.1016/j.jhep.2016.06.015

9. Kozbial K, Moser S, Schwarzer R, et al. Unexpected high incidence of hepatocellular carcinoma in cirrhotic patients with sustained virologic response following interferon-free direct-acting antiviral treatment. J Hepatol. 2016;65:856-858. doi:10.1016/j.jhep.2016.06.009

10. Cardoso H, Vale AM, Rodrigues S, et al. High incidence of hepatocellular carcinoma following successful interferon-free antiviral therapy for hepatitis C associated cirrhosis. $J$ Hepatol. 2016;65:10701071. doi:10.1016/j.jhep.2016.07.027

11. Romano A, Angeli P, Piovesan S, et al. Newly diagnosed hepatocellular carcinoma in patients with advanced hepatitis $\mathrm{C}$ treated with DAAs: a prospective population study. $J$ Hepatol. 2018;69:345-352. doi:10.1016/j.jhep.2018.03.009

12. Waziry R, Hajarizadeh B, Grebely J, et al. Hepatocellular carcinoma risk following direct-acting antiviral HCV therapy: a systematic review, meta-analyses, and meta-regression. $J$ Hepatol. 2017;67:1204-1212. doi:10.1016/j.jhep.2017.07.025

13. Cabibbo G, Petta S, Calvaruso V, et al. Is early recurrence of hepatocellular carcinoma in HCV cirrhotic patients affected by treatment with direct-acting antivirals? A prospective multicentre study. Aliment Pharmacol Ther. 2017;46:688-695. doi:10.1111/apt.14256

14. Ioannou G, Green P, Berry K. HCV eradication induced by directacting antiviral agents reduces the risk of hepatocellular carcinoma. $J$ Hepatol. 2018;68:25-32. doi:10.1016/j.jhep.2017.08.030

15. Saraiya N, Yopp AC, Rich NE, et al. Systematic review with metaanalysis: recurrence of hepatocellular carcinoma following directacting antiviral therapy. Aliment Pharmacol Ther. 2018;48:127-137. doi:10.1111/apt.14823

16. Guarino M, Viganò L, Ponziani FR, et al. Recurrence of hepatocellular carcinoma after direct acting antiviral treatment for hepatitis $\mathrm{C}$ virus infection: literature review and risk analysis. Dig Liver Dis. 2018;50:1105-1114. doi:10.1016/j.dld.2018.08.001

17. Ioannou GN, Beste LA, Green PK, et al. Increased risk for hepatocellular carcinoma persists up to 10 years after $\mathrm{HCV}$ eradication in patients with baseline cirrhosis or high FIB-4 scores. Gastroenterology. 2019;157:1264-1278. doi:10.1053/j.gastro.2019.07.033

18. Singal AG, Rich NE, Mehta N, et al. Direct-acting antiviral therapy not associated with recurrence of hepatocellular carcinoma in a multicenter North American Cohort Study. Gastroenterology. 2019;156:1683-1692. doi:10.1053/j.gastro.2019.01.027

19. Sapena V, Enea M, Torres F, et al. Hepatocellular carcinoma recurrence after direct-acting antiviral therapy: an individual patient data meta-analysis. Gut. 2021;gutjnl-2020-323663. doi:10.1136/gutjnl2020-323663

20. Calvaruso V, Cabibbo G, Cacciola I, et al. Incidence of hepatocellular carcinoma in patients with $\mathrm{HCV}$-associated cirrhosis treated with direct-acting antiviral agents. Gastroenterology. 2018;155:411-421. doi:10.1053/j.gastro.2018.04.008

21. Nahon P, Layese R, Bourcier V, et al. Incidence of hepatocellular carcinoma after direct antiviral therapy for $\mathrm{HCV}$ in patients with cirrhosis included in surveillance programs. Gastroenterology. 2018;155:1436-1450. doi:10.1053/j.gastro.2018.07.015

22. Degasperi E, D'Ambrosio R, Iavarone M, et al. Factors associated with increased risk of de novo or recurrent hepatocellular carcinoma in patients with cirrhosis treated with direct-acting antivirals for $\mathrm{HCV}$ infection. Clin Gastroenterol Hepatol. 2019;17:1183-1191. doi:10.1016/j.cgh.2018.10.038

23. ANRS collaborative study group on hepatocellular carcinoma (ANRS CO22 HEPATHER, CO12 CirVir and CO23 CUPILT cohorts). Lack of evidence of an effect of direct-acting antivirals on the recurrence of hepatocellular carcinoma: data from three ANRS cohorts. $J$ Hepatol. 2016;65:734-740. doi:10.1016/j.jhep.2016.05.045 
24. European Association for the Study of the Liver. EASL recommendations on treatment of hepatitis C: final update of the series. $J$ Hepatol. 2020;73:1170-1218.

25. Ravaioli F, Conti F, Brillanti S, et al. Hepatocellular carcinoma risk assessment by the measurement of liver stiffness variation in HCV cirrhotics treated with direct acting antivirals. Dig Liv Dis. 2018;50:573-579. doi:10.1016/j.dld.2018.02.010

26. Degasperi E, Galmozzi E, Facchetti F, et al. TLL1 variants do not predict hepatocellular carcinoma development in HCV cirrhotic patients treated with direct-acting antivirals. $J$ Viral Hepat. 2019;26:1233-1236. doi:10.1111/jvh.13155

27. Rinaldi L, Guarino M, Perrella A, et al. Role of liver stiffness measurement in predicting HCC occurrence in direct-acting antivirals setting: a real-life experience. Dig Dis Sci. 2019;64:3013-3019. doi:10.1007/s10620-019-05604-8

28. Lleo A, Aglitti A, Aghemo A, et al. Predictors of hepatocellular carcinoma in HCV cirrhotic patients treated with direct acting antivirals. Dig Liv Dis. 2019;51:310-317. doi:10.1016/j.dld.2018.10.014

29. Casadei Gardini A, Foschi FG, Conti F, et al. Immune inflammation indicators and ALBI score to predict liver cancer in HCV-patients treated with direct-acting antivirals. Dig Liver Dis. 2019;51:681-688. doi:10.1016/j.dld.2018.09.016

30. Bergna I, Degasperi E, D’Ambrosio R. Suboptimal accuracy of GES score to stratify post-SVR HCC risk in a single center cohort of European cirrhotics infected with any HCV genotype. Liver Int. 2021;41:1152-1153. doi:10.1111/liv.14700

31. Degasperi E, Galmozzi E, Pelusi S, et al. Hepatic Fat-Genetic Risk score predicts hepatocellular carcinoma in cirrhotic patients treated with DAAs. Hepatology. 2020;72:1912-1923. doi:10.1002/hep.31500

32. Sangiovanni A, Alimenti E, Gattai R, et al. Undefined/non-malignant nodules are associated with early occurrence of HCC in DAA-treated patients with HCV-related cirrhosis. J Hepatol. 2020;73:593-602. doi:10.1016/j.jhep.2020.03.030

33. Fan R, Papatheodoridis G, Sun J, et al. aMAP risk score predicts hepatocellular carcinoma development in patients with chronic hepatitis C. J Hepatol. 2020;73:1368-1378. doi:10.1016/j.jhep.2020.07.025

34. Kanwal F, Kramer J, Asch SM, et al. Risk of hepatocellular cancer in $\mathrm{HCV}$ patients treated with direct-acting antiviral agents. Gastroenterology. 2017;153:996-1005. doi:10.1053/j. gastro.2017.06.012

35. Finkelmeier F, Dultz G, Peiffer KH, et al. Risk of de-novo hepatocellular carcinoma after HCV treatment with direct-acting antivirals. Liver Cancer. 2018;7:190-204. doi:10.1159/000486812

36. Rinaldi L, Perrella A, Guarino M, et al. Incidence and risk factors of early $\mathrm{HCC}$ occurrence in $\mathrm{HCV}$ patients treated with direct-acting antivirals: a prospective multicenter study. $J$ Transl Med. 2019;17:292. doi:10.1186/s12967-019-2033-x

37. Abe K, Wakabayashi H, Nakayama H, et al. Factors associated with hepatocellular carcinoma occurrence after $\mathrm{HCV}$ eradication in patients without cirrhosis or with compensated cirrhosis. PLoS One. 2020;12:e243473. doi:10.1371/journal.pone.0243473

38. Ogawa E, Furusyo N, Nomura H, et al. Short-term risk of hepatocellular carcinoma after hepatitis $\mathrm{C}$ virus eradication following directacting anti-viral treatment. Aliment Pharmacol Ther. 2018;47:104113. doi:10.1111/apt.14380

39. Pinero F, Mendizabal M, Ridruejo E, et al. Treatment with directacting antivirals for HCV decreases but does not eliminate the risk of hepatocellular carcinoma. Liver Int. 2019;30:1033-1043. doi:10.1111/liv.14041

40. Ide T, Koga H, Nakano M, et al. Direct-acting antiviral agents do not increase the incidence of hepatocellular carcinoma development: a prospective, multicenter study. Hepatol Intern. 2019;13:293-301. doi:10.1007/s12072-019-09939-2

41. Kanwal F, Kramer JR, Asch SM, et al. Long-term risk of hepatocellular carcinoma in HCV patients treated with direct acting antiviral agents. Hepatology. 2020;71:44-55. doi:10.1002/hep.30823
42. Tanaka Y, Ogawa E, Huang CF, et al. HCC risk post-SVR with DAAs in East Asians: findings from the REAL-C cohort. Hepatol Intern. 2020;14:1023-1033. doi:10.1007/s12072-020-10105-2

43. de Franchis R. Expanding consensus in portal hypertension: report of the Baveno VI Consensus Workshop: stratifying risk and individualizing care for portal hypertension. J Hepatol. 2015;63:743-752. doi:10.1016/j.jhep.2015.05.022

44. Shiha G, Waked I, Soliman R, et al. GES: a validated simple score to predict the risk of HCC in patients with HCV-GT4-associated advanced liver fibrosis after oral antivirals. Liver Int. 2020;40:2828-2833. doi:10.1111/liv.14666

45. Alonso Lopez S, Manzano ML, Gea F, et al. A model based on noninvasive markers predicts very low hepatocellular carcinoma risk after viral response in hepatitis C virus - advanced fibrosis. Hepatology. 2020;72:1924-1934. doi:10.1002/hep.31588

46. Pons M, Rodriguez-Tajes S, Esteban JI, et al. Non-invasive prediction of liver-related events in patients with $\mathrm{HCV}$-associated compensated advanced chronic liver disease after oral antivirals. $J$ Hepatol. 2020;72:472-480. doi:10.1016/j.jhep.2019.10.005

47. Iio E, Matsuura K, Shimada N, et al. TLL1 variant associated with development of hepatocellular carcinoma after eradication of hepatitis C virus by interferon-free therapy. J Gastroenterol. 2019;54:339346. doi:10.1007/s00535-018-1526-3

48. Tamaki N, Higuchi M, Kurosaki M, et al. Risk assessment of hepatocellular carcinoma development by magnetic resonance elastography in chronic hepatitis $\mathrm{C}$ patients who achieved sustained virological responses by direct-acting antivirals. $J$ Viral Hepat. 2019;26:893-899. doi:10.1111/jvh.13103

49. Shiha G, Mikhail N, Soliman R. External validation of aMAP risk score in chronic hepatitis C genotype 4 patients with liver cirrhosis who achieved SVR following DAAs. J Hepatol. 2021;74:994-996. doi:10.1016/j.jhep.2020.10.008

50. Watanabe T, Tokumoto Y, Joko K, et al. Predictors of hepatocellular carcinoma occurrence after direct-acting antiviral therapy in patients with hepatitis C virus infection. Hepatol Res. 2019;49:136-146. doi:10.1111/hepr.13278

51. Kwon JH, Yoo SH, Nam SW, et al. Clinical outcomes after the introduction of direct antiviral agents for patients infected with genotype $1 \mathrm{~b}$ hepatitis $\mathrm{C}$ virus depending on the regimens: a multicenter study in Korea. J Med Virol. 2019;91:1104-1111. doi:10.1002/ jmv. 25412

52. Tani J, Morishita A, Sakamoto T, et al. Simple scoring system for prediction of hepatocellular carcinoma occurrence after hepatitis $\mathrm{C}$ virus eradication by direct-acting antiviral treatment: all Kagawa Liver Disease Group Study. Oncol Lett. 2020;19:2205-2212. doi:10.3892/ol.2020.11341

53. Søholm J, Hansen JF, Mossner B, et al. Low incidence of HCC in chronic hepatitis $\mathrm{C}$ patients with pretreatment liver stiffness measurement below 17.5 kilopascal who achieve SVR following DAAs. PLoS One. 2020;15:e243725. doi:10.1371/journal.pone.0243725

54. Nagata H, Nakagawa M, Asahina Y, et al. Effect of interferon-based and -free therapy on early occurrence and recurrence of hepatocellular carcinoma in chronic hepatitis C. J Hepatol. 2017;67:933-939. doi:10.1016/j.jhep.2017.05.028

55. Ogawa E, Nomura H, Nakamuta M, et al. Development of hepatocellular carcinoma by patients aged 75-84 with chronic Hepatitis C treated with direct-acting antivirals. J Infect Dis. 2020;jiaa359. doi:10.1093/infdis/jiaa359

56. Tachi Y, Hirai T, Kojima Y, et al. Liver stiffness measurement predicts hepatocellular carcinoma development in patients treated with direct-acting antivirals. J Gastroenterol Hepatol. 2017;1:44-49.

57. Ogasawara N, Saitoh S, Akuta N, et al. Advantage of liver stiffness measurement before and after direct-acting antiviral therapy to predict hepatocellular carcinoma and exacerbation of esophageal varices in chronic hepatitis C. Hepatol Res. 2020;50:426-438. doi:10.1111/ hepr. 13467 
58. Higuchi M, Tamaki N, Kurosaki M, et al. Prediction of hepatocellular carcinoma after sustained virological response using magnetic resonance elastography. Clin Gastroenterol Hepatol. 2019;17:2616-2618. doi:10.1016/j.cgh.2018.11.046

59. Watanabe T, Tokumoto Y, Joko K, et al. Sex difference in the development of hepatocellular carcinoma after direct-acting antiviral therapy in patients with HCV infection. J Med Virol. 2020;92:35073515. doi:10.1002/jmv.25984

60. Hiraoka A, Kumada T, Ogawa C, et al. Proposed a simple score for recommendations of scheduled ultrasonography surveillance for hepatocellular carcinoma after direct acting antivirals: multicenter analysis. J Gastroenterol Hepatol. 2019;34:436-441. doi:10.1111/ jgh. 14378

61. Virlogeux V, Pradat P, Hartig-Lavie K, et al. Direct-acting antiviral therapy decreases hepatocellular carcinoma recurrence rate in cirrhotic patients with chronic hepatitis C. Liver Int. 2017;37:1122-1127. doi:10.1111/liv.13456

62. Ikeda K, Kawamura Y, Kobayashi M, et al. Direct-acting antivirals decreases tumor recurrence after initial treatment of hepatitis $\mathrm{C}$ virusrelated hepatocellular carcinoma. Dig Dis Sci. 2017;62:2932-2942. doi:10.1007/s10620-017-4739-z

63. Torres HA, Vauthey JN, Mahale P, et al. Hepatocellular carcinoma recurrence after treatment with direct-acting antivirals: first, do no harm by withdrawing treatment. $J$ Hepatol. 2016;65:856-868. doi:10.1016/j.jhep.2016.05.034

64. Kolly P, Waidmann O, Vermehren J, et al. Hepatocellular carcinoma recurrence after direct antiviral agent treatment: a European multicentre study. J Hepatol. 2017;67:876-888. doi:10.1016/j. jhep.2017.07.007

65. Kogiso T, Sagawa T, Kodama K, et al. Hepatocellular carcinoma after direct-acting antiviral drug treatment in patients with hepatitis $\mathrm{C}$ virus. JGH Open. 2018;3:52-60. doi:10.1002/jgh3.12105
66. Nakano M, Koga H, Ide T, et al. Predictors of hepatocellular carcinoma recurrence associated with the use of direct-acting antiviral agent therapy for hepatitis $\mathrm{C}$ virus after curative treatment: a prospective multicenter cohort study. Cancer Med. 2019;8:2646-2653. doi:10.1002/cam4.2061

67. Zou WY, Choi K, Kramer JR, et al. Risk of hepatocellular cancer recurrence in Hepatitis $\mathrm{C}$ virus+ patients treated with direct-acting antiviral agents. Dig Dis Sci. 2019;64:3328-3336. doi:10.1007/ s10620-019-05641-3

68. Ahn YH, Lee H, Kim DY, et al. Independent risk factors for hepatocellular carcinoma recurrence after direct-acting antiviral therapy in patients with chronic hepatitis C. Gut Liver. 2020. doi:10.5009/ gnl20151

69. Jin J, Zhu P, Liao Y, et al. Elevated preoperative aspartate aminotransferase to lymphocyte ratio index as an independent prognostic factor for patients with hepatocellular carcinoma after hepatic resection. Oncotarget. 2015;6:19217-19227. doi:10.18632 oncotarget. 4265

70. Chhatwal J, Wang X, Ayer T, et al. Hepatitis C disease burden in the United States in the era of oral direct-acting antivirals. Hepatology. 2016;64:1442-1450. doi:10.1002/hep.28571

71. World Health Organization. Guidelines for the screening care and treatment of persons with chronic hepatitis $\mathrm{C}$ infection: updated version; 2016. Available from: https://www.ncbi.nlm.nih.gov/books/ NBK362924/. Accessed June 5, 2021.

72. Zavaglia C, Okolicsanyi S, Cesarini L, et al. Is the risk of neoplastic recurrence increased after prescribing direct-acting antivirals for HCV patients whose HCC was previously cured? J Hepatol. 2017;66:236-251. doi:10.1016/j.jhep.2016.08.016
Journal of Hepatocellular Carcinoma

\section{Publish your work in this journal}

The Journal of Hepatocellular Carcinoma is an international, peerreviewed, open access journal that offers a platform for the dissemination and study of clinical, translational and basic research findings in this rapidly developing field. Development in areas including, but not limited to, epidemiology, vaccination, hepatitis therapy, pathology and molecular tumor classification and prognostication are all considered for publication. The manuscript management system is completely online and includes a very quick and fair peer-review system, which is all easy to use. Visit http://www.dovepress.com/ testimonials.php to read real quotes from published authors. 